\title{
PRIME ARITHMETIC TEICHMÜLLER DISCS IN $\mathcal{H}(2)$
}

\author{
PASCAL HUBERT AND SAMUEL LELIÈVRE
}

\begin{abstract}
It is well-known that Teichmüller discs that pass through "integer points" of the moduli space of abelian differentials are very special: they are closed complex geodesics. However, the structure of these special Teichmüller discs is mostly unexplored: their number, genus, area, cusps, etc.

We prove that in genus two all translation surfaces in $\mathcal{H}(2)$ tiled by a prime number $n>3$ of squares fall into exactly two Teichmüller discs, only one of them with elliptic points, and that the genus of these discs has a cubic growth rate in $n$.
\end{abstract}

Keywords: Teichmüller discs, square-tiled surfaces, Weierstrass points. MSC: 32G15 (37C35 30F30 14H55 30F35)

\section{Contents}

1. Introduction 1

2. Background 5

3. Specific Tools $\quad 12$

4. Results 15

5. Proof of main theorem (two orbits) 17

6. Proof of results about elliptic points 27

7. Proof of countings 29

8. Strong numerical evidence $\quad 35$

Appendix A. $n=3$ and $n=5 \quad 36$

Appendix B. Hyperelliptic components of other strata 37

Appendix C. The theorem of Gutkin and Judge 37

References 41

\section{INTRODUCTION}

In his fundamental paper of 1989, Veech studied the finite-volume Teichmüller discs. Translation surfaces with such discs, called Veech

Date: 26 October 2004. 
surfaces, enjoy very interesting dynamical properties: their directional flows are either completely periodic or uniquely ergodic. An abundant literature exists on Veech surfaces: Veech [Ve89, Ve92], Gutkin-Judge [GuJu1, GuJu2], Vorobets [Vo], Ward [Wa], KenyonSmillie [KeSm], Hubert-Schmidt [HuSc00, HuSc01], Gutkin-HubertSchmidt [GuHuSc], Calta [Ca], McMullen [Mc]...

The simplest examples of Veech surfaces are translation covers of the torus (ramified over a single point), called square-tiled surfaces. They are those translation surfaces whose stabilizer in $\mathrm{SL}(2, \mathbf{R})$ is arithmetic (commensurable with $\mathrm{SL}(2, \mathbf{Z})$ ), by a theorem of Gutkin and Judge. These surfaces (and many more!) were introduced by Thurston [Th] and studied on the dynamical aspect by Gutkin [Gu], Veech [Ve87] and Gutkin-Judge [GuJu1, GuJu2]. Square-tiled surfaces can be viewed as the "integer points" of the moduli spaces of holomorphic 1-forms. The asymptotic number of integer points in a large ball was used by Zorich [Zo] and Eskin-Okounkov [EsOk] to compute volumes of strata of abelian differentials.

It was known for years that Teichmüller discs passing through these integer points in the moduli space are very special: they are closed (complex) geodesics. Despite enormous interest to invariant submanifolds (especially to the simplest ones: those of complex dimension one), absolutely nothing was known about the structure of these special Teichmüller discs: about their number, genus, area, cusps, etc. It was neither known which "integer points" belong to the same Teichmüller disc.

1.1. Main results. In this paper, we study square-tiled surfaces in the stratum $\mathcal{H}(2)$. This stratum is the moduli space of holomorphic 1 -forms with a unique (double) zero on a surface of genus two. For surfaces tiled by a prime number of squares, we show:

Theorem 1.1. For any prime $n \geqslant 5$, the $\mathrm{SL}(2, \mathbf{R})$-orbits of $n$-squaretiled surfaces in $\mathcal{H}(2)$ form two Teichmüller discs $D_{A}(n)$ and $D_{B}(n)$.

Theorem 1.2. $D_{A}(n)$ and $D_{B}(n)$ can be seen as the unit tangent bundles to orbifold surfaces with the following asymptotic behavior:

- genus $\sim c n^{3}$, with $c_{A}=c_{B}=(3 / 16)(1 / 12)$,

- area $\sim c n^{3}$, with $c_{A}=c_{B}=(3 / 16)(\pi / 3)$, 
- number of cusps $\sim c n^{2}$, with $c_{A}=1 / 24$ and $c_{B}=1 / 8$,

- number of elliptic points $O(n)$, one of them having none.

Proposition 1.3. All these discs arise from L-shaped billiards.

Our results are extended by McMullen [Mc2] to describe the repartition into different orbits of all Veech surfaces in $\mathcal{H}(2)$. In particular, the invariant introduced in $\S 4.2$ also determines orbits in the nonprime case.

1.2. Side results. We find the following as side results of our study:

- One-cylinder directions.

Proposition 1.4. All surfaces in $\mathcal{H}(2)$ tiled by a prime number of squares have one-cylinder directions i.e. directions in which they decompose into one single cylinder.

- Discs without elliptic points. During some time, the search for new Veech surfaces focused on examples arising from billiards in rational-angled polygons. Angles of the billiard table not multiples of the right angle lead to elliptic elements in the Veech group. Billiards with all angles multiples of the right angle have however recently been studied, especially L-shaped billiards (see [Mc]).

- Discs of (arbitrary high) positive genus. When a Veech group has positive genus, the subgroup generated by its parabolic elements has infinite index, and cannot be a lattice. This implies that the naive algorithm which consists in finding parabolic elements in the Veech group cannot lead to obtain the whole group not even up to finite index.

The surfaces arising from billiards in the regular polygons, studied by Veech in [Ve92], have genus tending to infinity, and one could probably show that the genus of their Veech groups also tends to infinity, though Veech does not state this explicitly.

Our examples give families of Teichmüller discs of arbitrarily high genus, the translation surfaces in these discs staying in genus two.

- Noncongruence subgroups. Since we deal with families of subgroups of $\operatorname{SL}(2, \mathbf{Z})$, it is natural to check whether they belong to the well-known family of congruence subgroups. Appendix A provides an example of a Veech group that is a non-congruence subgroup 
of SL $(2, \mathbf{Z})$. Another example was given by G. Schmithüsen [Schmi]. A detailed discussion of the congruence problem in this setting will appear in [HL].

- Deviation from the mean order.

Proposition 1.5. The number of n-square-tiled surfaces in $\mathcal{H}(2)$ for prime $n$ is asymptotically $1 / \zeta(4)$ times the mean order of the number of $n$-square-tiled surfaces in $\mathcal{H}(2)$.

1.3. Methods. We parametrize square-tiled surfaces in $\mathcal{H}(2)$ by using separatrix diagrams as in [KoZo], [Zo] and [EsMaSc]. These coordinates bring the study of Teichmüller discs of $n$-square-tiled surfaces down to a combinatorial problem.

We want to describe the $\operatorname{SL}(2, \mathbf{Z})$ orbits of these surfaces. Using the fact that $\mathcal{H}(2)$ is a hyperelliptic stratum, the combinatorial representation of Weierstrass points allows us to show there are at least two orbits for odd $n \geqslant 5$. Showing there are only two is done for prime $n$ in a combinatorial way, by a careful study of the action of generators of $\mathrm{SL}(2, \mathbf{Z})$ on square-tiled surfaces.

For the countings, we use generating functions.

1.4. Related works. Our counting results are very close to the formulae in [EsMaSc]. Eskin-Masur-Schmoll calculate Siegel-Veech constants for torus coverings in genus two. In $\mathcal{H}(2)$, these calculations are based on counting the square-tiled surfaces with a given number of squares. The originality of our work is to count squaretiled surfaces disc by disc.

There are also analogies with Schmoll's work [Schmo]. He computes the explicit Veech groups of tori with two marked points and the quadratic asymptotics for theses surfaces. Some of the methods he uses are intimately linked to those used in our work. The Veech groups he exhibits are all congruence subgroups.

A computer program allows to give all the geometric information on Teichmüller discs of square-tiled surfaces in $\mathcal{H}(2)$. Schmithüsen [Schmi] has a program to compute the Veech group of any given square-tiled surface. She also found positive genus discs as well as noncongruence Veech groups. Möller [Mö] computes algebraic equations of some square-tiled surfaces and of their Teichmüller curves. 
1.5. Acknowledgements. We thank Anton Zorich for stating questions and some conjectures. We thank the Institut de Mathématiques de Luminy and the Max-Planck-Institut für Mathematik for excellent welcome and working conditions. We thank Joël Rivat, Martin Schmoll and other participants of the conference 'Dynamique dans l'espace de Teichmüller et applications aux billards rationnels' at CIRM in 2003. We thank Martin Möller and Gabriela Schmithüsen for comments on a previous version of this paper, circulated under the title "Square-tiled surfaces in $\mathcal{H}(2)$ ".

\section{BACKGROUND}

2.1. Translation surfaces, Veech surfaces. Let $S$ be an oriented compact surface of genus $g$. A translation structure on $S$ consists in a set of points $\left\{P_{1}, \ldots, P_{n}\right\}$ and a maximal atlas on $S \backslash\left\{P_{1}, \ldots, P_{n}\right\}$ with translation transition functions.

A holomorphic 1-form $\omega$ on $S$ induces a translation structure by considering its natural parameters, and its zeros as points $P_{1}, \ldots, P_{n}$. All translation structures we consider are induced by holomorphic 1-forms. Slightly abusing vocabulary and notation, we refer to a translation surface $(S, \omega)$, or sometimes just $S$ or $\omega$.

A translation structure defines: a complex structure, since translations are conformal; a flat metric with cone-type singularities of angle $2\left(k_{i}+1\right) \pi$ at order $k_{i}$ zeros of the 1 -form; and directional flows $\mathcal{F}_{\theta}$ on $S$ for $\left.\left.\theta \in\right]-\pi, \pi\right]$.

Orbits of the flows $\mathcal{F}_{\theta}$ meeting singularities (backward, resp. forward) are called (outgoing, resp. incoming) separatrices in the direction $\theta$. Orbits meeting singularities both backward and forward are called saddle connections; the integrals of $\omega$ along them are the associated connection vectors.

Define the singularity type of a 1 -form $\omega$ to be the unordered tuple $\sigma=\left(k_{1}, \ldots, k_{n}\right)$ of orders of its zeros (recall $k_{1}+\ldots+k_{n}=2 g-2$, all $\left.k_{i}>0\right)$. The singularity type is invariant by orientation-preserving diffeomorphisms. The moduli space $\mathcal{H}_{g}$ of holomorphic 1 -forms on $S$ is the quotient of the set of translation structures by the group $\operatorname{Diff}^{+}(S)$ of orientation-preserving diffeomorphisms. $\mathcal{H}_{g}$ is stratified by singularity types, the strata are denoted by $\mathcal{H}(\sigma)$. 
$\operatorname{SL}(2, \mathbf{R})$ acts on holomorphic 1-forms: if $\omega$ is a 1-form, $\{(U, f)\}$ the translation structure given by its natural parameters, and $A \in$ $\mathrm{SL}(2, \mathbf{R})$, then $A \cdot \omega=\{(U, A \circ f)\}$. As is well known, this action (to the left) commutes with that (to the right) of $\operatorname{Diff}^{+}(S)$ and preserves singularity types. Each stratum $\mathcal{H}(\sigma)$ thus inherits an $\operatorname{SL}(2, \mathbf{R})$ action. The dynamical properties of this action have been extensively studied by Masur and Veech [Ma, Ve82, etc.].

From the behavior of the $\operatorname{SL}(2, \mathbf{R})$-orbit of $\omega$ in $\mathcal{H}(\sigma)$ one can deduce properties of directional flows $\mathcal{F}_{\theta}$ on the translation surface $(S, \omega)$. The Veech dichotomy expressed below is a remarkable illustration of this.

Call affine diffeomorphism of $(S, \omega)$ an orientation-preserving homeomorphism $f$ of $S$ such that the following three conditions hold - $f$ keeps the set $\left\{P_{1}, \ldots, P_{n}\right\}$ invariant;

- $f$ restricts to a diffeomorphism of $S \backslash\left\{P_{1}, \ldots, P_{n}\right\}$;

- the derivative of $f$ computed in the natural charts of $\omega$ is constant. The derivative can then be shown to be an element of $\operatorname{SL}(2, \mathbf{R})$.

Affine diffeomorphisms of $(S, \omega)$ form its affine group $\operatorname{Aff}(S, \omega)$, their derivatives form its Veech group $V(S, \omega)<\mathrm{SL}(2, \mathbf{R})$, a noncocompact fuchsian group. The Veech group is the stabilizer of $(S, \omega)$ for the action of $\operatorname{SL}(2, \mathbf{R})$ on $\mathcal{H}_{g}$. Veech showed that the derivation map $\operatorname{Aff}(S, \omega) \rightarrow V(S, \omega)$ is finite-to-one. We show (Proposition 4.4) that in $\mathcal{H}(2)$ it is one-to-one.

Theorem (Veech dichotomy). If $V(S, \omega)$ is a lattice in $\mathrm{SL}(2, \mathbf{R})$ (i.e. $\operatorname{vol}(V(S, \omega) \backslash \mathrm{SL}(2, \mathbf{R}))<\infty)$ then for each direction $\theta$, either the flow $\mathcal{F}_{\theta}$ is uniquely ergodic, or all orbits of $\mathcal{F}_{\theta}$ are compact and $S$ decomposes into a finite number of cylinders of commensurable moduli.

Cylinder decompositions are further discussed in $\S 2.3$. Translation surfaces with lattice Veech group are called Veech surfaces.

2.2. Square-tiled surfaces, lattice of periods. A translation covering is a map $f:\left(S_{1}, \omega_{1}\right) \longrightarrow\left(S_{2}, \omega_{2}\right)$ of translation surfaces that

- is topologically a ramified covering;

- maps zeros of $\omega_{1}$ to zeros of $\omega_{2}$;

- is locally a translation in the natural parameters of $\omega_{1}$ and $\omega_{2}$. 
Translation covers of the standard torus marked at the origin are the simplest examples of Veech surfaces. Such surfaces are tiled by squares.

We call them square-tiled. The Gutkin-Judge theorem states:

Theorem (Gutkin-Judge). A translation surface $(S, \omega)$ is squaretiled if and only if its Veech group $V(S, \omega)$ shares a finite-index subgroup with $\mathrm{SL}(2, \mathbf{Z})$.

Translation surfaces with such (arithmetic) Veech groups have also been called arithmetic; another name for them is origamis. A proof of Gutkin and Judge's theorem, very different from the original, is given in appendix $\mathrm{C}$.

The subgroup of $\mathbf{R}^{2}$ generated by connection vectors is the lattice of relative periods of $(S, \omega)$, denoted by $\Lambda(\omega)$.

Lemma 2.1. A translation surface $(S, \omega)$ is square-tiled if and only if $\Lambda(\omega)$ is a rank 2 sublattice of $\mathbf{Z}^{2}$.

Proof. If $(S, \omega)$ is square-tiled, connection vectors are obviously integer vectors, so they span a sublattice of $\mathbf{Z}^{2}$. Conversely, let

$f:(S, \omega) \rightarrow \mathbf{R}^{2} / \Lambda(\omega), \quad$ where $z_{0}$ is a given point of $(S, \omega)$. $z \quad \mapsto \int_{z_{0}}^{z} \omega \bmod \Lambda(\omega)$,

The integral is well-defined modulo the lattice of absolute periods; $f$ is a fortiori well-defined. Since $f$ is holomorphic and onto, it is a covering. Since relative periods are integer-valued, it is clear that zeros of $\omega$ project to the origin. So, given a point $P \neq 0$ on the torus, preimages of $P$ are all regular points, so $P$ is not a branch point. Hence the covering is ramified only above the origin. Composing $f$ with the covering $g: \mathbf{R}^{2} / \Lambda(\omega) \longrightarrow \mathbf{R}^{2} / \mathbf{Z}^{2}$, we see $(S, \omega)$ is squaretiled.

A square-tiled surface $(S, \omega)$ is called primitive if $\Lambda(\omega)=\mathbf{Z}^{2}$.

Lemma 2.2. Let $(S, \omega)$ be an n-square-tiled surface of genus $g>1$. If $n$ is prime then $\Lambda(\omega)=\mathbf{Z}^{2}$.

Proof. Lemma 2.1 shows that $(S, \omega)$ is a ramified cover of $\mathbf{R}^{2} / \Lambda(\omega)$. Let $d$ be the degree of the covering. Then $n=d \cdot\left[\mathbf{Z}^{2}: \Lambda(\omega)\right]$. So obviously if $n$ is prime then $\Lambda(\omega)=\mathbf{Z}^{2}$. 

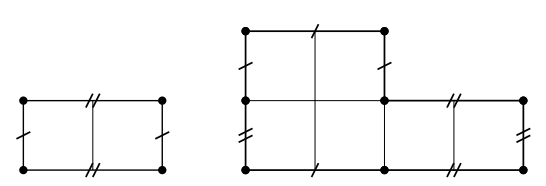

Note that $\Lambda(\omega)$ is not always $\mathbf{Z}^{2}$, as shown by the examples in the figure. On the left, a torus $T$ with lattice of periods $2 \mathbf{Z} \times \mathbf{Z}$ and Veech group generated by $\left(\begin{array}{ll}1 & 2 \\ 0 & 1\end{array}\right)$ and $\left(\begin{array}{cc}1 & 0 \\ 1 / 2 & 1\end{array}\right)$. On the right, a genus 2 cover of $T$, with $\Lambda(\omega)=2 \mathbf{Z} \times \mathbf{Z}$ and Veech group generated by $\left(\begin{array}{ll}1 & 4 \\ 0 & 1\end{array}\right)$ and $\left(\begin{array}{cc}0 & -2 \\ 1 / 2 & 0\end{array}\right)$.

The following lemma was explained to us first by Martin Schmoll then by Anton Zorich.

Lemma 2.3. Let $(S, \omega)$ be a square-tiled surface, then $V(S, \omega)$ is a subgroup in $V\left(\mathbf{R}^{2} / \Lambda(\omega), \mathrm{d} z\right)$. In particular, if $(S, \omega)$ is primitive, then $V(S, \omega)<\mathrm{SL}(2, \mathbf{Z})$.

Proof. Let $\phi$ :

$$
\begin{array}{ccc}
V(S, \omega) & \rightarrow & V\left(\mathbf{R}^{2} / \Lambda(\omega), \mathrm{d} z\right), \\
A=d f, f \in \operatorname{Aff}(S, \omega) & \mapsto & A .
\end{array}
$$

The only difficulty is to show that $\phi$ is well-defined i.e. that any element $A$ in $V(S, \omega)$ preserves $\Lambda(\omega)$. Since any element of the affine group maps a connection to a connection, hence $A$ maps a connection vector to a connection vector (i.e. an element in $\Lambda(\omega)$ ).

Remark. As shown by the examples above, there are Veech groups of square-tiled surfaces which are not subgroups of $\operatorname{SL}(2, \mathbf{Z})$.

2.3. Cylinders of square-tiled surfaces. A square-tiled surface decomposes into maximal horizontal cylinders, bounded above and below by unions of saddle connections, each of which appears once on the top of a cylinder and once on the bottom of a cylinder. Gluing the cylinders alongs these saddle connections builds back the surface.

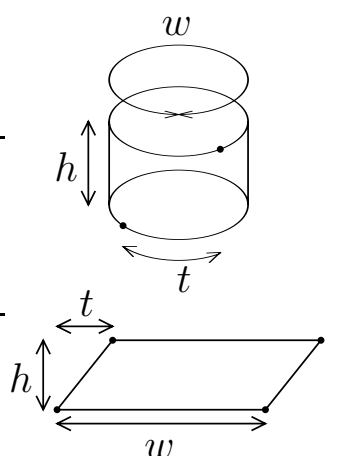
cylinder, for instance some ends of saddle connections. to $\mathbf{R} / w \mathbf{Z} \times[0, h]$, for some $h$ and $w$.

A cylinder on a translation surface is isometric

Convention. We refer to these dimensions as height and width respectively, whether the 'horizontal direction of the cylinder' coincides with the horizontal direction of the surface or not.

An additional twist parameter $t$ is needed, measuring the distance along the 'horizontal direction of the cylinder' between some (arbitrary) reference points on the bottom and top of the 


\subsection{Action of $\operatorname{SL}(2, \mathbf{Z})$ on square-tiled surfaces.}

Lemma 2.4. The $\mathrm{SL}(2, \mathbf{Z})$-orbit of a primitive $n$-square-tiled surface is the set of primitive $n$-square-tiled surfaces in its $\mathrm{SL}(2, \mathbf{R})$-orbit.

Proof. SL $(2, \mathbf{Z})$ preserves $\mathbf{Z}^{2}(=\Lambda(\omega)$ if $(S, \omega)$ is primitive squaretiled) and hence the property of being primitive square-tiled. Conversely, if $(S, \omega)$ is primitive square-tiled and $\left(S_{1}, \omega_{1}\right)=A \cdot(S, \omega)$ is square-tiled for some $A \in \operatorname{SL}(2, \mathbf{R})$, then $\Lambda\left(\omega_{1}\right)=A \cdot \Lambda(\omega)$ means $A$ preserves $\mathbf{Z}^{2}$, so $A \in \mathrm{SL}(2, \mathbf{Z})$.

Remark. The number of squares, $n$, is preserved by $\mathrm{SL}(2, \mathbf{R})$ because it is the area of the surface. Consequently $\mathrm{SL}(2, \mathbf{Z}) \cdot(S, \omega)$ is finite.

Notation. Denote by $U=\left(\begin{array}{ll}1 & 1 \\ 0 & 1\end{array}\right)$ and $R=\left(\begin{array}{cc}0 & -1 \\ 1 & 0\end{array}\right)$ the standard generators of $\operatorname{SL}(2, \mathbf{Z})$, and by $\mathcal{U}=\langle U\rangle=\left\{\left(\begin{array}{ll}1 & n \\ 0 & 1\end{array}\right): n \in \mathbf{Z}\right\}$ the subgroup generated by $U$. Here is the action of $U \stackrel{+}{\rightarrow} \stackrel{U}{\rightarrow}+\underset{+}{\text { and } R \text { on squares. }}$

The action on square-tiled surfaces is obtained by applying the same to all square tiles. The new horizontal cylinder decomposition is then recovered by cutting and gluing (see example in $\S 3.4$ ).

2.5. Hyperelliptic surfaces, Weierstrass points. Recall that a Riemann surface $X$ of genus $g$ is hyperelliptic if there exists a degree 2 meromorphic function on $X$. Such a function induces a holomorphic involution on $X$. This involution has $2 g+2$ fixed points called Weierstrass points. The set of these points is invariant by all automorphisms of the complex structure. A translation surface is called hyperelliptic if the underlying Riemann surface is hyperelliptic.

Hyperelliptic translation surfaces have been studied by Veech. He showed [Ve95] that in genus $g$ they are obtained from centrosymmetric polygons with $4 g$ or $4 g+2$ sides by pairwise identifying opposite sides.

The hyperelliptic involution is in these coordinates the reflection in the center of the polygon; the Weierstrass points are the center of the polygon, the midpoints of its sides, and the vertices (identified into one point) in the $4 g$ case (in the $4 g+2$ case the vertices are indentified into two points exchanged by the hyperelliptic involution). 
2.6. Cusps. Let $\Gamma$ be a fuchsian group. A parabolic element of $\Gamma$ is a matrix of trace 2 (or -2 ). A point of the boundary at infinity of $\mathbf{H}^{2}$ is parabolic if it is fixed by a parabolic element of $\Gamma$. A cusp is a conjugacy class under $\Gamma$ of primitive parabolic elements (primitive meaning not powers of other parabolic elements of $\Gamma$ ).

Recall that a lattice admits only a finite number of cusps.

Geometrically, each cusp in $\Gamma \backslash \mathbf{H}^{2}$ has, for some positive $\lambda$ called its width, neighborhoods isometric to the quotients of the strips $\{z \in \mathbf{C}: 0<|\operatorname{Re} z|<\lambda, \operatorname{Im} z>M\}$ by the translation $z \mapsto z+\lambda$, for large $M$.

On a Veech surface $(S, \omega)$, any 'periodic' direction is fixed by a parabolic element of the Veech group. Conversely the eigendirection of a parabolic element in the Veech group is a 'periodic' direction. We call such directions parabolic. Thus parabolic limit points of $V(S, \omega)$ are cotangents of parabolic directions.

When $(S, \omega)$ is a square-tiled surface, the set of parabolic limit points is $\mathbf{Q}$. Cusps are therefore equivalence classes of rationals under the homographic action of $V(S, \omega)$. The following lemma gives a combinatorial description of cusps for a square-tiled surface.

Lemma 2.5 (Zorich). Let $(S, \omega)$ be a primitive $n$-square-tiled surface and $E=\mathrm{SL}(2, \mathbf{Z}) \cdot(S, \omega)$ the set of n-square-tiled surfaces in its orbit. The cusps of $(S, \omega)$ are in bijection with the $\mathcal{U}$-orbits of $E$.

Proof. Denote by $\mathcal{C}$ the set of cusps of $(S, \omega)$.

$$
\begin{aligned}
& \text { Let } \varphi: \quad \mathrm{SL}(2, \mathbf{Z}) \stackrel{f}{\rightarrow} \quad \mathrm{Q} \quad \stackrel{\pi}{\rightarrow} \quad \mathcal{C} \text {, } \\
& A \quad \mapsto A^{-1} \infty \mapsto A^{-1} \infty \bmod V(S, \omega) .
\end{aligned}
$$

Note that $\infty$ corresponds to the horizontal direction in $(S, \omega)$ because the projective action is the action on co-slopes and not on slopes. $A^{-1} \infty$ corresponds to the direction on $(S, \omega)$ that is mapped by $A$ to the horizontal direction of $A \cdot(S, \omega)$.

$\varphi$ pulls down as $\psi: \quad E \quad \rightarrow \quad \mathcal{C}$,

$$
A \cdot(S, \omega) \mapsto A^{-1} \infty \bmod V(S, \omega) .
$$

$\psi$ is well-defined: if $A \cdot(S, \omega)=B \cdot(S, \omega)$, then $\exists P \in V(S)$, $B=A P$, so setting $A^{-1} \infty=\alpha, B^{-1} \infty=\beta$, we have $\beta=B^{-1} \infty=$ $\left(B^{-1} A\right) A^{-1} \infty=P^{-1} \alpha$, so $\alpha$ and $\beta$ correspond to the same cusp. Further, $\psi$ is surjective because $f$ is. Indeed, $\forall \alpha=p / q, \exists A \in \operatorname{SL}(2, \mathbf{Z})$ s.t. $A^{-1} \infty=\alpha$. (The orbit of $\infty$ under $\operatorname{SL}(2, \mathbf{Z})$ is $\mathbf{Q}$.) 
Recall that the stabilizer of $\infty$ for the action of $\operatorname{SL}(2, \mathbf{Z})$ is $\mathcal{U}$. If $\psi\left(S_{1}, \omega_{1}\right)=\psi\left(S_{2}, \omega_{2}\right)$, where $\left(S_{1}, \omega_{1}\right)=A \cdot(S, \omega)$ and $\left(S_{2}, \omega_{2}\right)=$ $B \cdot(S, \omega)$, then $\varphi(A)=\varphi(B)$.

Let $\alpha=f(A)=A^{-1} \infty$ and $\beta=f(B)=B^{-1} \infty$. Since $\alpha$ and $\beta$ correspond to the same cusp, $\exists P \in V(S)$ s.t. $\beta=P \alpha$. So $\infty=A \alpha=$ $A P^{-1} \beta=A P^{-1} B^{-1} \infty$ which implies $A P^{-1} B^{-1} \in \mathcal{U}$ i.e. $\exists U^{k} \in \mathcal{U}$ s.t. $A P^{-1}=U^{k} B$ i.e. $A P^{-1} \cdot(S, \omega)=A \cdot(S, \omega)=U^{k} B \cdot(S, \omega)$, so that $\left(S_{1}, \omega_{1}\right)$ and $\left(S_{2}, \omega_{2}\right)$ are in the same $\mathcal{U}$-orbit.

Conversely: if $\left(S_{2}, \omega_{2}\right)=U^{k}\left(S_{1}, \omega_{1}\right)$ with $U^{k} \in \mathcal{U}$, and $\left(S_{2}, \omega_{2}\right)=$ $B \cdot(S, \omega)$ and $\left(S_{1}, \omega_{1}\right)=A \cdot(S, \omega)$, then $\psi\left(S_{2}, \omega_{2}\right)=B^{-1} \infty=$ $A^{-1} U^{-k} \infty=A^{-1} \infty=\psi\left(S_{1}, \omega_{1}\right)$.

2.7. Elliptic points. Recall that in a fuchsian group $\Gamma$, any elliptic element has finite order and is conjugate to a rational rotation.

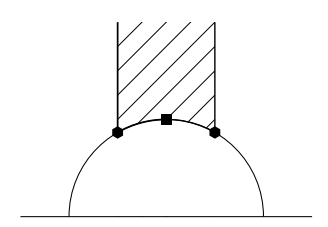

A fixed point in $\mathbf{H}^{2}$ of an elliptic element of $\Gamma$ is called elliptic. Its projection to the quotient $\Gamma \backslash \mathbf{H}^{2}$ is a cone point, with a curvature default. For instance the modular surface $\mathrm{SL}(2, \mathbf{Z}) \backslash \mathbf{H}^{2}$ has two cone points, of angles $\pi$ and $2 \pi / 3$.

Suppose that $\Gamma$ is the Veech group of a translation surface and has an elliptic point. By applying a convenient element of $\mathrm{SL}(2, \mathbf{R})$, we can suppose that this point is $i$. The corresponding elliptic element is a rational rotation. The translation surfaces which project to $i$ have this rotation in their Veech group. This roughly means that they have an apparent symmetry. At the Riemann surface level, the rotation is an automorphism of the complex structure (it modifies the vertical direction but not the metric). For genus 1 , the cone point $i$ (resp. $e^{i \pi / 3}$ ) of the modular surface corresponds to the square (resp. hexagonal) torus, which has a symmetry of projective order 2 (resp. $3)$.

One should note that the translation surfaces obtained from rational polygonal billiards always have elliptic elements in their Veech group: writing the angles of a simple polygon as $\left(k_{1} \pi / r, \ldots, k_{q} \pi / r\right)$, with $k_{1}, \ldots, k_{q}, r$ coprime, the covering translation surface is obtained by gluing $2 r$ copies by symmetry. The rotation of angle $2 \pi / r$ is in the Veech group (this rotation is minus the identity if $r=2$ ). Many explicit calculations of lattice Veech groups make use of this remark (see [Ve89], [Vo], [Wa]). Our method is completely different. 
2.8. The Gauss-Bonnet Formula. Let $\Gamma$ be a finite-index subgroup of $\operatorname{SL}(2, \mathbf{Z})$ containing - Id. The quotient of $\Gamma \backslash \mathrm{SL}(2, \mathbf{R})$ is the unit tangent bundle to an orbifold surface with cusps $S_{\Gamma}$. Algebraic information on the group is related to the geometry of the surface.

Let $d$ be the index $[\operatorname{SL}(2, \mathbf{Z}): \Gamma]$ of $\Gamma$ in $\operatorname{SL}(2, \mathbf{Z}), e_{2}$ (resp. $\left.e_{3}\right)$ the number of conjugacy classes of elliptic elements of order 2 (resp. 3) of $\Gamma, e_{\infty}$ the number of conjugacy classes of cusps of $\Gamma$.

Then the surface $S_{\Gamma}$ has hyperbolic area $d \frac{\pi}{3}, e_{2}$ cone points of angle $\pi, e_{3}$ cone points of angle $\frac{2 \pi}{3}, e_{\infty}$ cusps, and its genus $g$ is given by:

The Gauss-Bonnet Formula. $g=1+d / 12-e_{2} / 4-e_{3} / 3-e_{\infty} / 2$.

\section{Specific Tools}

In this section we give specific properties of the stratum $\mathcal{H}(2)$, and a combinatorial coordinate system for square-tiled surfaces in $\mathcal{H}(2)$.

3.1. Hyperellipticity. First recall that any genus 2 Riemann surface is hyperelliptic. Given a genus 2 Riemann surface $X$ and its hyperelliptic involution $\tau$, any 1 -form $\omega$ on $X$ satisfies $\tau^{*} \omega=-\omega$.

In the moduli space of holomorphic 1 -forms of genus $2, \mathcal{H}(2)$ is the stratum of 1 -forms with a degree 2 zero (a cone point of angle $6 \pi$ ).

As said in $\S 2.5$, any translation surface in $\mathcal{H}(2)$ can be represented as a centro-symmetric octagon. The six Weierstrass points are the center of the polygon, the middles of the sides and the cone-type singularity. The position of the Weierstrass points in a surface decomposed into horizontal cylinders is described in $\S 5.1$.

3.2. Separatrix diagrams. Forms in $\mathcal{H}(2)$ have a single degree 2 zero, geometrically a cone point of angle $6 \pi$, with three outgoing separatrices and three incoming ones in any direction.

Recall that the horizontal direction of a square-tiled surface is completely periodic; the horizontal separatrices are saddle connections. The combinatorics of these connections is called a separatrix diagram in $[\mathrm{KoZ}$ ]. The surface is obtained from this diagram by gluing cylinders along the saddle connections.

Each outgoing horizontal separatrix returns to the saddle making an angle $\pi, 3 \pi$ or $5 \pi$ with itself. Four separatrix diagrams are combinatorially possible (up to rotation by $2 \pi$ around the cone point); 
they correspond to return angles $(\pi, \pi, \pi),(\pi, 3 \pi, 5 \pi),(3 \pi, 3 \pi, 3 \pi)$, $(5 \pi, 5 \pi, 5 \pi)$ :
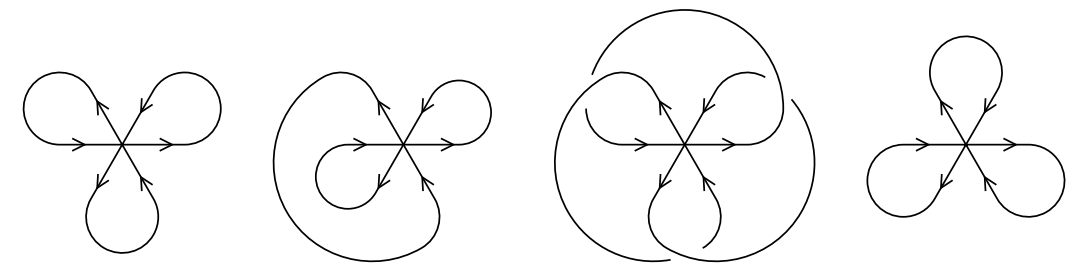

There is no consistent way of gluing cylinders along the saddle connections of the first and last diagrams to obtain a translation surface.

The second diagram is possible with the condition that the saddle connections that return with angles $\pi$ and $5 \pi$ have the same length; this diagram corresponds to surfaces with two cylinders. The third diagram corresponds to surfaces with one cylinder, with no restriction on the lengths of the saddle connections.

3.3. Parameters for square-tiled surfaces in $\mathcal{H}(2)$. Here we give complete combinatorial coordinates for square-tiled surfaces in $\mathcal{H}(2)$. See figures in $\S 5.1$.

Notation. We use $\wedge$ for greatest common divisor, and $\vee$ for least common multiple.

3.3.1. One-cylinder surfaces. A one-cylinder surface is parametrized by the height of the cylinder, the lengths of the three horizontal saddle connections (a triple of integers up to cyclic permutation), and the twist parameter. If all three horizontal saddle connections have the same length, the twist parameter is taken to be less than that length; otherwise, less than the sum of the three lengths.

For primitive surfaces, the height is 1 , and the lengths of the three horizontal saddle connections add up to the area $n$ of the surface.

The horizontal saddle connections appear in some (cyclic) order on the bottom of the cylinder, and in reverse order on the top.

3.3.2. Two-cylinder surfaces. Labeling the horizontal saddle connections according to their return angles, call them $\gamma_{\pi}, \gamma_{3 \pi}, \gamma_{5 \pi}$. Call $\ell_{1}$ the common length of $\gamma_{\pi}$ and $\gamma_{5 \pi}$, and $\ell_{2}$ the length of $\gamma_{3 \pi}$. One cylinder is bounded below by $\gamma_{\pi}$ and above by $\gamma_{5 \pi}$; the other one is bounded below by $\gamma_{5 \pi}$ and $\gamma_{3 \pi}$, and above by $\gamma_{\pi}$ and $\gamma_{3 \pi}$. 
A two-cylinder surface is determined by the heights $h_{1}, h_{2}$ and widths $w_{1}=\ell_{1}, w_{2}=\ell_{1}+\ell_{2}>w_{1}$ of the cylinders as well as two twist parameters $t_{1}, t_{2}$ satisfying $0 \leqslant t_{1}<w_{1}, 0 \leqslant t_{2}<w_{2}$. The area of the surface is $h_{1} w_{1}+h_{2} w_{2}=n$. For primitive surfaces, $h_{1} \wedge h_{2}=1$. For prime $n$, in addition, $\ell_{1} \wedge \ell_{2}=1$, and $(\mathrm{P}) \ell_{1} \wedge h_{2}=1$.

3.4. Action of $\operatorname{SL}(2, \mathbf{Z})$. The action of $R$ (rotation by $\pi / 2$ ) does not preserve separatrix diagrams in general. The horizontal cylinder decomposition of $R \cdot S$ is the vertical cylinder decomposition of $S$.

$U$ is the primitive parabolic element in $\mathrm{SL}(2, \mathbf{Z})$ that preserves the horizontal direction. Its action preserves separatrix diagrams, as well as heights $h_{i}$ and widths $w_{i}$ of horizontal cylinders $C_{i}$, and only changes twist parameters $t_{i}$ to $\left(t_{i}+h_{i}\right) \bmod w_{i}$.

Here is an example of how $U$ acts on a surface.
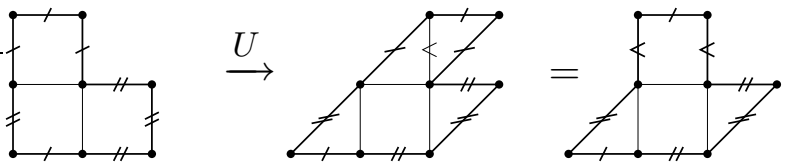

For prime $n$, given a cyclically ordered 3-partition $(a, b, c)$ of $n$, all one-cylinder surfaces with bottom sides of lengths $a, b, c$ (up to cyclic permutation) are in the same $\mathcal{U}$-orbit, or cusp (see Lemma 2.5).

The following lemma describes $U$-orbits of two-cylinder surfaces in $\mathcal{H}(2)$ by giving their sizes and canonical representatives.

Lemma 3.1. Let $S$ be a primitive two-cylinder n-square-tiled surface in $\mathcal{H}(2)$ with parameters $h_{i}, w_{i}, t_{i}(i=1,2)$. Then the cardinality of its $U$-orbit (its cusp width) is

$\mathrm{cw}(S)=\frac{w_{1}}{w_{1} \wedge h_{1}} \vee \frac{w_{2}}{w_{2} \wedge h_{2}} \quad\left(=\frac{w_{1}}{w_{1} \wedge h_{1}} \times \frac{w_{2}}{w_{2} \wedge h_{2}}\right.$ for prime $\left.n\right)$.

The surface $S^{\prime}$ with $h_{i}^{\prime}=h_{i}, w_{i}^{\prime}=w_{i}$, and $t_{i}^{\prime}=t_{i} \bmod \left(w_{i} \wedge h_{i}\right)$ is a "canonical" representative of the $U$-orbit of $S$. Each surface thus has a unique representative with $0 \leqslant t_{i}^{\prime}<w_{i} \wedge h_{i}$.

Proof. Observe that $U^{k} \cdot S$ has widths $w_{i}$, heights $h_{i}$, and twist parameters $\left(t_{i}+k h_{i}\right) \bmod w_{i}$. So for $U^{k} \cdot S$ to coincide with $S$, the integer $k$ must be a multiple of $\frac{w_{i}}{w_{i} \wedge h_{i}}$ for each $i$. The cusp width is the least such positive $k$, the least common multiple of $\frac{w_{1}}{w_{1} \wedge h_{1}}$ and $\frac{w_{2}}{w_{2} \wedge h_{2}}$. The second part is a simple application of the Chinese remainder theorem. 


\section{Results}

This section expands the results summarized in the introduction, detailed proofs are postponed to the next sections. Additional conjectures appear in $\S 8$.

4.1. Two orbits. Theorem 1.1 can be reformulated as:

Proposition 4.1. Given a prime $n \geqslant 5$, the primitive $n$-square-tiled surfaces in $\mathcal{H}(2)$ fall into two $\mathrm{SL}(2, \mathbf{Z})$ orbits.

The idea for proving this is first to give an invariant which takes two different values, thus proving that there are at least two orbits (see $\S 4.2$ below, and $\S 5.1$ ), then prove that there are exactly two orbits by showing that each orbit contains a one-cylinder surface (see $\S 5.2)$, and that all one-cylinder surfaces with the same invariant are indeed in the same orbit $(\S 5.3)$.

We will call these orbits $\mathrm{A}$ and $\mathrm{B}$.

Remark. An extension of this result in some components of higherdimensional strata is presented in appendix B.

4.2. Invariant. We present a geometric invariant that can easily be computed for any primitive square-tiled surface in $\mathcal{H}(2)$ (for instance presented in its decomposition into horizontal cylinders.)

The Weierstrass points of a surface in $\mathcal{H}(2)$ are

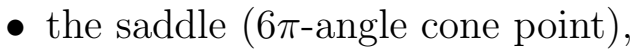

- and five regular points.

Lemma 4.2. The number of integer Weierstrass points of a primitive square-tiled surface is invariant under the action of $\mathrm{SL}(2, \mathbf{Z})$.

By integer point we mean a vertex of the square tiling. The proof of the lemma is obvious, since $\operatorname{SL}(2, \mathbf{Z})$ preserves $\mathbf{Z}^{2}$.

Proposition 4.3. Primitive $n$-square-tiled surfaces in $\mathcal{H}(2)$ have

- for $n=3$, exactly 1 integer Weierstrass point,

- for even $n$, exactly 2 ,

- for odd $n$, either 1 or 3 (both values occur).

Martin Möller pointed out to us that this invariant also appears in $[\mathrm{Ka}, \S 2$, formula (6)] in algebraic geometric language; Kani's normalized covers correspond to our orbit B. This invariant is also mentioned in [Mö, Remark 3.4]. 


\subsection{Elliptic affine diffeomorphisms.}

Proposition 4.4. A translation surface in $\mathcal{H}(2)$ has no nontrivial translation in its affine group. Hence the derivation from its affine group to its Veech group is an isomorphism.

Proposition 4.5. A translation surface in $\mathcal{H}(2)$ can have no elliptic element of order 3 in its Veech group.

Lemma 4.6. Any $R$-invariant Veech surface in $\mathcal{H}(2)$ can be represented as a $R$-invariant octagon.

Proposition 4.7. For any given prime $n$, there exist $R$-invariant $n$-square-tiled $\mathcal{H}(2)$ surfaces. All of them have the same invariant, namely, $A$ if $n \equiv-1$ [4] and $B$ if $n \equiv 1$ [4].

Remark. This proposition implies the following interesting fact: there are finite-covolume Teichmüller discs with no elliptic points. This differs from the billiard case which has been the main source of explicit examples of lattice Veech groups.

4.4. Countings. The asymptotic number of square-tiled surfaces in $\mathcal{H}(2)$ of area bounded by $N$ is given in [Zo] (see also [EsOk] and [EsMaSc]) to be $\zeta(4) \frac{N^{4}}{24}$ for one-cylinder surfaces and $\frac{5}{4} \zeta(4) \frac{N^{4}}{24}$ for two-cylinder surfaces. The mean order for the number of squaretiled surfaces of area exactly $n$ is therefore $\zeta(4) \frac{n^{3}}{6}$ for one-cylinder surfaces and $\frac{5}{4} \zeta(4) \frac{n^{3}}{6}$ for two-cylinder surfaces.

The following proposition, from which Theorem 1.2 follows, states that for prime $n$, there are in fact asymptotics for these numbers, which are $\zeta(4)$ times smaller than the mean order.

Proposition 4.8. For prime $n$, there are $O(n)$ elliptic points, and the following countings and asymptotics hold for surfaces and cusps, according to the number of cylinders and to the orbit.

surfaces:

cusps:

\begin{tabular}{|c|c|c|c||c|c|c|c|}
\hline & $1-c y l$ & $2-c y l$ & all & & $1-c y l$ & $2-c y l$ & all \\
\hline$A$ & $\frac{n(n-1)(n+1)}{24}$ & $\sim \frac{7}{8} \frac{n^{3}}{6}$ & $\sim \frac{9}{8} \frac{n^{3}}{6}$ & $A$ & $\frac{(n-1)(n+1)}{24}$ & $o\left(n^{3 / 2+\varepsilon}\right)$ & $\sim \frac{n^{2}}{24}$ \\
\hline$B$ & $\frac{n(n-1)(n-3)}{8}$ & $\sim \frac{3}{8} \frac{n^{3}}{6}$ & $\sim \frac{9}{8} \frac{n^{3}}{6}$ & $B$ & $\frac{(n-1)(n-3)}{8}$ & $o\left(n^{3 / 2+\varepsilon}\right)$ & $\sim \frac{n^{2}}{8}$ \\
\hline all & $\frac{n(n-1)(n-2)}{6}$ & $\sim \frac{5}{4} \frac{n^{3}}{6}$ & $\sim \frac{9}{4} \frac{n^{3}}{6}$ & all & $\frac{(n-1)(n-2)}{6}$ & $o\left(n^{3 / 2+\varepsilon}\right)$ & $\sim \frac{n^{2}}{6}$ \\
\hline
\end{tabular}


This proposition gives more detail than Theorem 1.2 by distinguishing one-cylinder and two-cylinder cusps and surfaces. Proposition 1.4 and Proposition 1.5 are corollaries of this proposition.

Remarks. Orbits A and B have asymptotically the same size (same number of square-tiled surfaces). However orbit B has asymptotically three times as many one-cylinder surfaces as orbit A.

In each orbit the proportion of two-cylinder cusps is asymptotically negligible; however it is not the case for the proportion of two-cylinder surfaces. This shows that the average width of the two-cylinder cusps grows faster than $n$. (One-cylinder cusps all have width $n$.)

\section{Proof of main theorem (TWo orbits)}

In this section we first prove Proposition 4.3, then Proposition 4.1.

Convention for figures. In all figures, we represent a square-tiled surface $S$ in $\mathcal{H}(2)$ by a fundamental octagonal domain. $S$ is obtained by identifying pairs of parallel sides of same length; all vertices (black dots) get identified to the saddle. Circles are sometimes used to indicate the other Weierstrass points.

Except in $\S 6.3 .2$, the octagon reflects horizontal cylinders: nonhorizontal sides are identified by horizontal translations. On one-cylinder surfaces, the horizontal sides on the top and on the bottom of the cylinder are identified in opposite cyclic order. Two-cylinder surfaces are represented

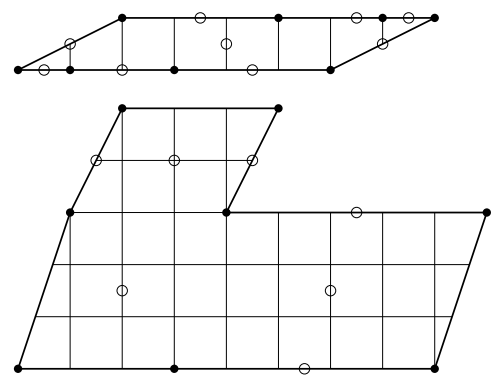
with the cylinder of least width on top of the widest one, to the left. Its top side is glued to the leftmost side under the bottom cylinder. The remaining two sides, to the right on the top and bottom of the bottom cylinder, are identified with each other.

5.1. Two values of the invariant. Here we prove Proposition 4.3, about the possible values of the number of integer Weierstrass points of a primitive square-tiled surface in $\mathcal{H}(2)$.

Recall that the hyperelliptic involution turns the cylinders upsidedown. We deduce the position of Weierstrass points (see figure). 
The saddle is always an integer Weierstrass point. We discuss the case of the remaining five, depending on the parity of the parameters.

Under the hyperelliptic involution:

- saddle connections that bound a cylinder both on its top and on its bottom are mapped to themselves with reversed orientation, so that their middpoint is fixed: it is a Weierstrass point, integer when the length of the saddle connection is even.

- the core circle of a cylinder, also mapped to itself with orientation reversed, has two antipodal fixed points. If the cylinder has odd height, none of them is integer. When the height is even and the width odd, one of them is integer. When the height and width are even, either both or none is integer, depending on the parity of the twist parameter.

5.1.1. One-cylinder case. The core of the (height 1) cylinder contains two non-integer Weierstrass points. The remaining three are the midpoints of the horizontal connections (whose lengths add up to $n$ ).

If $n$ is odd, it splits into either 3 odd lengths (no integer Weierstrass point), or 1 odd and 2 even lengths (2 integer Weierstrass points). For $n=3$ all lengths are 1 (hence odd); for greater odd $n$ both cases occur.

If $n$ is even, two lengths are odd and one even (if all were even, the surface could not be primitive). This completes the one-cylinder case.

5.1.2. Two-cylinder case. We use parameters $h_{1}, h_{2}, w_{1}, w_{2}, t_{1}, t_{2}$ introduced above. We also use $\ell_{1}$ and $\ell_{2}$ to denote the lengths of the horizontal saddle connections. We then have:

$\ell_{1}=w_{1}, \ell_{1}+\ell_{2}=w_{2}, n=w_{1} h_{1}+w_{2} h_{2}=h_{1} \ell_{1}+h_{2}\left(\ell_{1}+\ell_{2}\right)(*)$.

- Odd $n$. If $\ell_{2}$ is even, the corresponding Weierstrass point is integer. Because $n$ is odd, equation (*) implies that $\ell_{1}$ is odd, thus both cylinders have odd widths, and still by $(*)$ one of the heights must be even. The corresponding cylinder has one integer Weierstrass point on its core line. The total number of integer Weierstrass points is then 3 .

If $\ell_{2}$ is odd, the corresponding Weierstrass point is non-integer; if $\ell_{1}$ is odd (resp. even), then $w_{2}$ is even (resp. odd), thus by $(*) h_{1}$ (resp. $h_{2}$ ) has to be odd, meaning the top (resp. bottom) cylinder contains 
two non-integer Weierstrass points. The two Weierstrass points in the bottom (resp. top) cylinder are integer if $h_{2}$ is even and $t_{2}$ is odd (resp. if $h_{1}$ and $t_{1}$ are even), non-integer otherwise (see figure above). The value of the invariant is accordingly 3 or 1 .

For $n=3, \ell_{1}=\ell_{2}=1$; for greater odd $n$ both values do occur.

- Even $n$. Recall that primitivity implies $h_{1} \wedge h_{2}=1$. In particular at least one of them is odd.

If both heights are odd, the Weierstrass points inside the cylinders are non-integer, and because $n=\left(h_{1}+h_{2}\right) \ell_{1}+h_{2} \ell_{2}$ is even, $\ell_{2}$ has to be even, so the last Weierstrass point is integer, and the invariant is 2 .

If $h_{1}$ is odd and $h_{2}$ even, then, by $(*), \ell_{1}$ has to be even. Then if $\ell_{2}$ is odd, the corresponding Weierstrass point is non-integer, one of the Weierstrass points inside the bottom cylinder is integer, and the invariant is 2 . If $\ell_{2}$ is even, the corresponding Weierstrass point is integer, and $t_{2}$ has to be odd for the surface to be primitive, hence the remaining Weierstrass points are non-integer, and the invariant is 2 .

The last case to consider is when $h_{1}$ is even and $h_{2}$ odd. If $\ell_{1}$ is odd, then so is $\ell_{2}$ (by $\left.(*)\right)$, so one Weierstrass point in the top cylinder is integer, and the invariant is 2 . If $\ell_{1}$ is even, then $\ell_{2}$ is also even by $(*)$. The corresponding Weierstrass point is integer, and $t_{1}$ is odd for primitiveness. Thus all Weierstrass points inside cylinders are non-integer, and the invariant is 2 .

This completes the two-cylinder case, and Proposition 4.3 is proved.

- Summary of two-cylinder case. For future reference, we sum up the case study above in a table giving the invariant for odd $n$ according to the parity of $h_{1}, h_{2}, \ell_{1}, \ell_{2}$ (recall that $w_{1}=\ell_{1}$ and $\left.w_{2}=\ell_{1}+\ell_{2}\right)$.

\begin{tabular}{ccccc}
\hline$h_{1}$ & $h_{2}$ & $\ell_{1}$ & $\ell_{2}$ & invariant \\
\hline 0 & 1 & 1 & 0 & 3 \\
1 & 0 & 1 & 0 & 3 \\
0 & 1 & 0 & 1 & $t_{1}$ odd: $1 ; t_{1}$ even: 3 \\
1 & 0 & 1 & 1 & $t_{2}$ odd: $3 ; t_{2}$ even: 1 \\
1 & 1 & 0 & 1 & 1 \\
1 & 1 & 1 & 1 & 1 \\
\hline
\end{tabular}

Table for odd $n$ case.

The other combinations of parities of the parameters cannot happen for odd $n$ and primitive surfaces.

Note that for even $n$ we concluded that the invariant is 2 for all primitive surfaces. 


\subsection{Reduction to one cylinder.}

Proposition 5.1. Each orbit contains a one-cylinder surface.

Equivalently, each surface has a direction in which it decomposes in one single cylinder.

A baby version of this proposition is the following lemma.

Lemma 5.2. A two-cylinder surface of height 2 tiled by a prime number of squares has one-cylinder directions.

Proof of the lemma. Consider a surface made of two cylinders, both of height 1 . Since $n$ is prime, the two widths are relatively prime. By acting by $U$, the twists can be set to any values (see Lemma 3.1). Set the top twist to 0 and the bottom twist to 1 . Then by considering the vertical flow, we get a one-cylinder surface.

We prove the proposition by induction on the height of the surface: given a two-cylinder surface, we show that its orbit contains a surface of strictly smaller height.

Consider a two-cylinder square-tiled surface $S$ in $\mathcal{H}(2)$, with a prime number of square tiles. By acting by $U$ we can move to the canonical representative of the same cusp (see Lemma 3.1), so we will assume $t_{i}<w_{i}, i=1,2$.

We split our study into four cases according to which twists are zero.

Case 1. Both twists are nonzero.

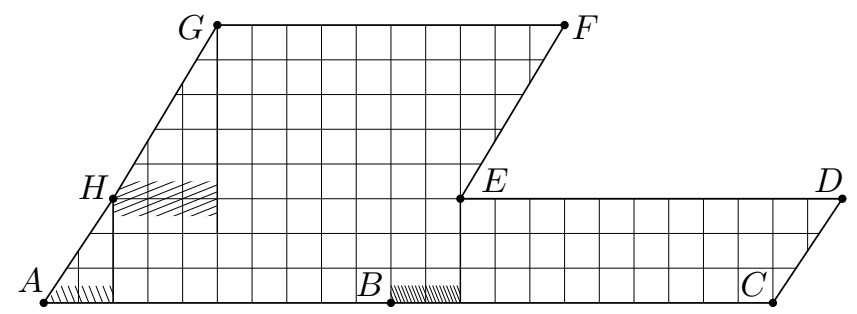

Call $h_{1}, h_{2}$ the heights and $t_{1}, t_{2}$ the twists of the horizontal cylinders of $S$. Consider the rotated surface $R S$. If $R S$ consists of one horizontal cylinder, we are done. Otherwise, it has two horizontal cylinders, which are the vertical cylinders of $S$, and fill $S$. Looking to the right of $A, H$, and $B$, we see all vertical cylinders of $S$. The vertical cylinder to the right of $A$ has height at most $t_{2}$, that to the 
right of $B$ also, and that to the right of $H$ at most $t_{1}$. So one of the vertical cylinders has heights at most $t_{2}$, and the other one has height at most $t_{1}$. The sum of their heights is hence at most $t_{1}+t_{2}$, so it is less than $h_{1}+h_{2}$.

Case 2. The bottom twist is nonzero but the top twist is zero.

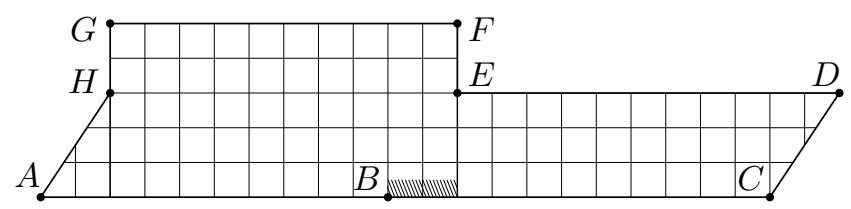

In this case the same vertical cylinder is to the right of $A$ and $H$. If the vertical separatrix going down from $H$ ends in $B$, there is only one vertical cylinder (one horizontal cylinder for the rotated surface $R S$ ); if not, it necessarily crosses the shaded region to the right of $B$, so there are two vertical cylinders, and the sum of their heights is at most $t_{2}$ (the twist of the bottom cylinder of $S$ ), hence less than the height of the bottom cylinder of $S$.

Case 3. The bottom twist is zero but the top twist is nonzero.

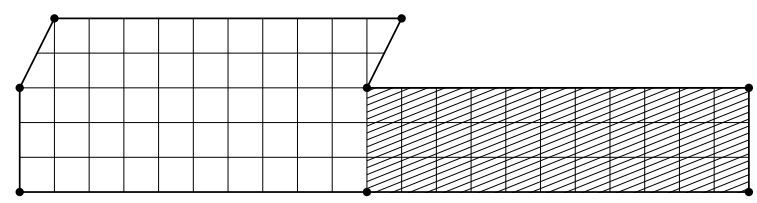

Act by $R$; this rotates $S$ by $\pi / 2$. The rotated surface $R \cdot S$ has two cylinders: a top cylinder, corresponding to the side part of $S$ (shaded on the figure), with twist 0 , and a bottom cylinder cylinder of height at most $t_{1}$, which we assumed to be less than $h_{1}$. The surface in the same cusp with least nonnegative twists also has top twist 0 , so if it has bottom twist 0 , conclude by case 4 , otherwise apply case 2 to obtain a surface of height less than $h_{1}$.

Case 4. The twist parameters are both zero. In this case we end the induction by jumping to a one-cylinder surface directly:

Lemma 5.3. The diagonal direction for the "base rectangle" of an $L$ surface tiled by a prime number of squares is a one-cylinder direction. 

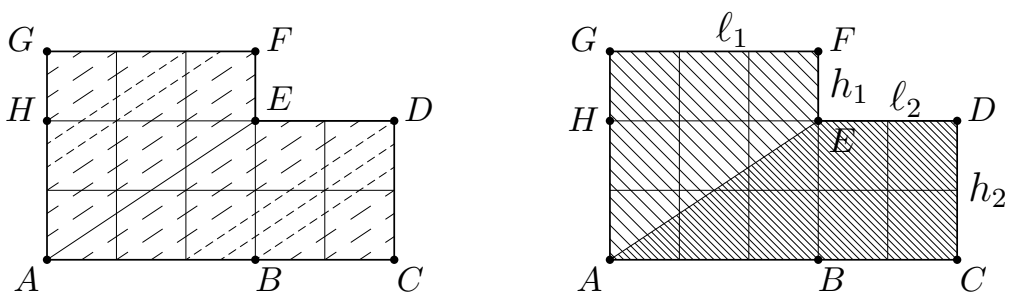

Proof. The ascending diagonal $[A E]$ of the base rectangle of our $\mathrm{L}$ surface cuts it into two zones. Note that $[A E]$ has no other integer point than $A$ and $E$ by $(\mathrm{P})$ of $\S 3.3 .2$.

The other two saddle connections parallel to $[A E]$ start from $B$ and $H$ and end in $F$ and $D$. We want to prove that the one starting from $H$ ends in $F$ and the one issued from $B$ ends in $D$, meaning each saddle connection returns with angle $3 \pi$.

Set the origin in $A$ or $E$ and consider coordinates modulo $\ell_{1} \mathbf{Z} \times h_{2} \mathbf{Z}$.

Follow a saddle connection parallel to $[A E]$ from integer point to integer point. While it winds in a same zone, the coordinates of the integer points it reaches remain constant modulo $\ell_{1} \mathbf{Z} \times h_{2} \mathbf{Z}$. Changing zone has the following effects for the coordinates of the next integer point:

- from the upper to the lower zone: decrease $y$ by $h_{1}$ modulo $h_{2}$;

- from the lower to the upper zone: decrease $x$ by $\ell_{2}$ modulo $\ell_{1}$.

Zone changes have to be alternated. Once inside a zone with the right coordinates modulo $\ell_{1} \mathbf{Z} \times h_{2} \mathbf{Z}$, a separatrix reaches the top right corner of the zone with no more zone change.

So we want to prove that starting from $B$, in the lower zone with coordinates $(0,0)$, and adding in turn $\left(-\ell_{2}, 0\right)$ and $\left(0,-h_{1}\right)$, coordinates $\left(\ell_{2}, 0\right)$ (point $\left.D\right)$ will be reached before $\left(0, h_{1}\right)$ (point $H$ ).

After $k$ changes from lower to upper zone and $k$ changes from upper to lower zone, the coordinates are final if $k \equiv-1\left[\ell_{1}\right]$ and $k \equiv 0\left[h_{2}\right]$; that is, if $k$ is $h_{2}\left(\ell_{1}-1\right)$. After $k+1$ changes from lower to upper zone and $k$ changes from upper to lower zone, the coordinates are final if $k \equiv 0\left[\ell_{1}\right]$ and $k \equiv 0\left[h_{2}\right]$, which means $k$ is $h_{2} \cdot \ell_{1}$. So the separatrix parallel to $[A E]$ starting from $B$ reaches $D$.

5.3. Linking one-cylinder surfaces of each type. We call a surface type A (resp. B) if it has 1 (resp. 3) integer Weierstrass points. 
Recall that a primitive one-cylinder surface in $\mathcal{H}(2)$ has height one, hence it is determined by the cyclically ordered lengths of the three saddle connections on the bottom of this cylinder (which add up to $n$ ), and by a twist parameter.

The repeated action of $U$ can set the twist parameter to any of its $n$ possible values, so for the purpose of linking surfaces of the same type by $\mathrm{SL}(2, \mathbf{Z})$ action, we may already consider surfaces with the same cyclically ordered partition $(a, b, c)$ as equivalent (allowing implicit $U$-action). We will call them $(a, b, c)$ surfaces.

Partitions into three odd numbers correspond to type A; partitions into two even numbers and one odd number correspond to type B.

We will first show that any one-cylinder surface has a $(1, *, *)$ surface in its orbit; then we will show that $(1, b, c)$ surfaces with $b$ and $c$ odd are in the orbit of a $(1,1, n-2)$ surface, proving all type A surfaces to be in one orbit; then that $(1,2 a, 2 b)$ surfaces are in the orbit of a $(1,2, n-3)$ surface, proving all type B surfaces to be in one orbit.

Consider a rational-slope direction on a square-tiled surface $S$; this direction is completely periodic. Say it is given by a vector $(p, q) \in$ $\mathbf{Z}^{2}$, with $p \wedge q=1$. For any $(u, v) \in \mathbf{Z}^{2}$ such that $\operatorname{det}\left(\begin{array}{cc}p & u \\ q & v\end{array}\right)=1$ our surface can be seen as tiled by parallelograms of sides $(p, q),(u, v)$, whose vertices are the vertices of the square tiling.

These parallelograms are taken to unit squares by $M=\left(\begin{array}{cc}p & u \\ q & v\end{array}\right)^{-1} \in$ $\mathrm{SL}(2, \mathbf{Z})$. We call $M \cdot S$ "the surface seen in direction $(p, q)$ " on $S$.

Consider a saddle connection $\sigma$ on $S$ in direction $(p, q)$; the corresponding saddle connection on $M \cdot S$ is horizontal with an integer length equal to the number of integer points (vertices of the square tiling) $\sigma$ reaches on $S$. Abusing vocabulary we also call this the length of $\sigma$.

A saddle connection returns at an angle of $3 \pi$ if and only if it has a Weierstrass point in its middle. If two saddle connections in a given direction return with angle $3 \pi$ then so does the third, and that direction is one-cylinder; thus two saddle connection lengths give the third.

5.3.1. First step: any one-cylinder surface has a $(1, *, *)$ surface in its orbit. To show this, we prove that an $(a, b, c)$ surface has a $(\delta, k \delta, \gamma)$ surface in its orbit, where $\delta \mid a \wedge b$. Then because $n$ is prime we have 
$\gamma \wedge \delta=1$, hence applying the argument a second time with $\gamma$ and $\delta$ in place of $a$ and $b$ shows that there is a $(1, *, *)$ one-cylinder surface in the orbit of the surface we started with.

The proof is as follows. Consider the $(a, b, c)$ surface $S$ having saddle connections of lengths $a, b, c$ on the bottom, $b, a, c$ on the top.

$R S$ has two cylinders, the top one of height $c$ and width 1 , and the bottom one of height $d=a \wedge b$ and width $\frac{a+b}{d}$, and some twist $t$.

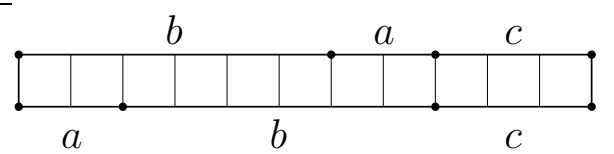

Now the direction $(1+t, d)$ is a $(\delta, k \delta, \gamma)$ one-cylinder direction with $\delta=(1+t) \wedge d$. Note that $k=\frac{a+b}{d}-1$, and that $\gamma \wedge \delta=1$.

So by applying this procedure twice we see that any surface has a $(1, *, *)$ one-cylinder surface in its orbit.

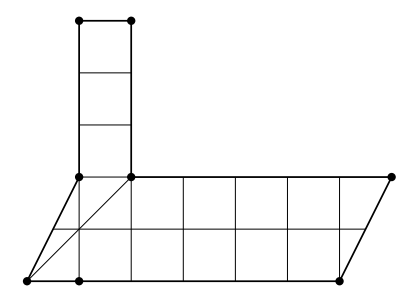

5.3.2. End of proof for type A surfaces. There only remains to link any $(1, b, c)$ surface, where $b$ and $c$ are odd, to a $(1,1, n-2)$ surface.

Consider the L surface with arms of width 1 and lengths $b$ and $c$.

Apply $U^{2}$ to set the bottom twist to 2 . Then rotate by applying $R$, and obtain a surface with two cylinders of height $1 . \quad$ By applying a convenient

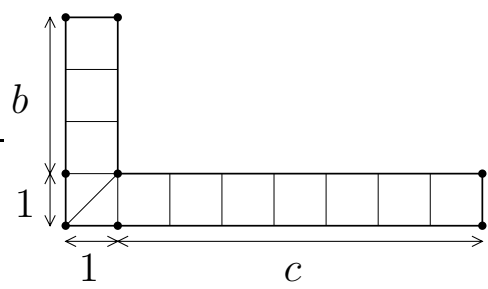
power of $U$ the twists can be made both 0 .

In the diagonal direction of the base rectangle of this new L surface, we see a $(1,1, n-2)$ surface.

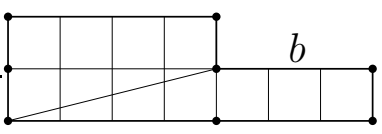

5.3.3. End of proof for type B surfaces. Here we take the one-cylinder surface with the partition $(1,2, n-3)$ as the reference surface, and prove by steps that any type B surface has it in its orbit.

To do this, we first show that any one-cylinder surface has a onecylinder surface with a $(1,2 a, 2 b)$ partition in its orbit. This is done by the first step explained above. 
Then we link

- $(1,2 a, 2 b)$ where $a \neq b$ with $(d, 2 d, *)$, then with $(1,2, n-3)$;

- $(1,2 a, 2 b)$ where $a=b$ with $(2,2, n-4)$, then with $(1,2, n-3)$.

- Linking $(1,2 a, 2 b)$ with $(1,2, *)$ when $a \neq b$.

Without loss of generality, suppose $a<b$. Consider the onecylinder surface with saddle connections of lengths $2 a, 2 b, 1$ on the bottom and $2 b, 2 a, 1$ on the top.

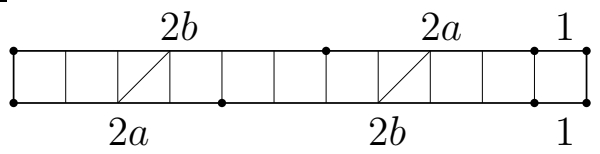

In the direction $(b-a, 1)$ there is a connection between two integer Weierstrass points, so in this direction we see a two-cylinder surface. Its top cylinder has height $2 a$ and width 2 and its bottom cylinder has height 1 and with $2+\ell$ for some $\ell$.

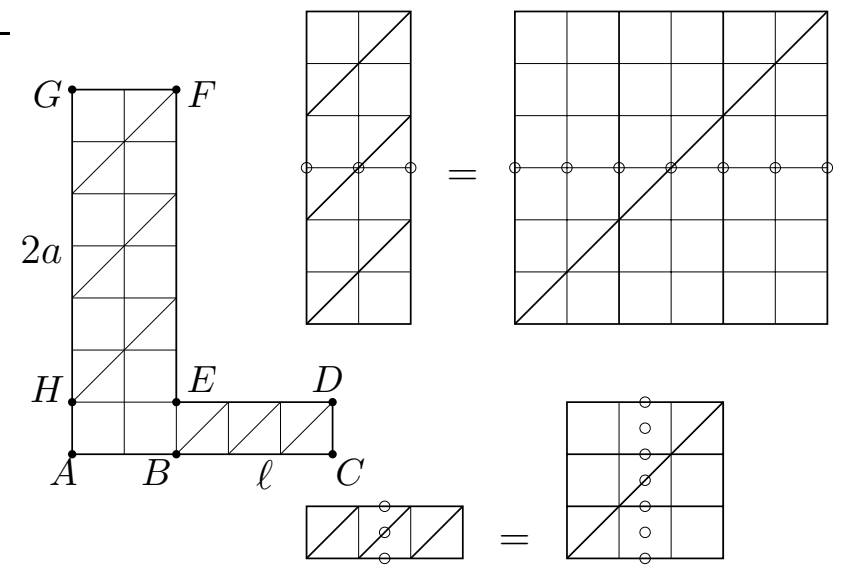

In certain directions, the separatrix issued from $H$ winds around the horizontal cylinder $H E G F$. In particular, in any direction $(k, a)$, $k \in \mathbf{N}$, it will run into a Weierstrass point (and into a saddle after twice the distance).

Likewise, in appropriate directions, the separatrix issued from $B$ winds around the vertical cylinder $B C D E$. In particular, in any direction $(\ell / 2, k / 2)$ (equivalently $\ell, k), k \in \mathbf{N}$, it will run into a Weierstrass point (and into a saddle after twice the distance).

Consider therefore the direction $(\ell, a)$. In this direction we get a $(d, 2 d, *)$ one-cylinder surface, where $d=a \wedge \ell$. 
Now there only remains to link $(d, 2 d, *)$ with $(1,2, *)$, which is easily done: consider the one-cylinder surface with saddle connections $d, 2 d, c$ on the bottom and $2 d, d, c$ on the top;

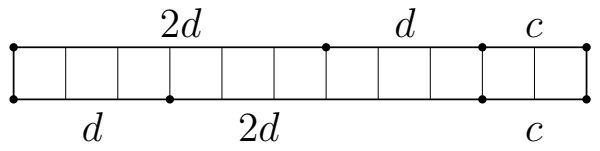

in the $(d, 1)$ direction we get a $(1,2, *)$ one-cylinder surface.

- Linking $(1,2 a, 2 b)$ with $(1,2, *)$ when $a=b$.

Consider the one-cylinder surface with saddle connections of length $2 a, 2 b, c$ on the bottom and $2 b, 2 a, c$ on the top.

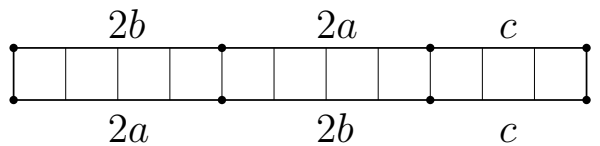

In the direction $(a, 1)$ we see a $(2,2, *)$ one-cylinder surface.

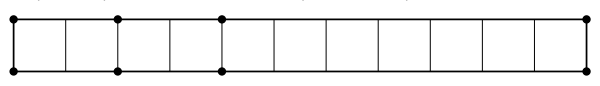

On this surface, in the direction $(2,1)$, we have a two-cylinder surface with its top cylinder of height 2 and width 1 , and its bottom cylinder of height 1 . Acting by $U$ we can set the twist parameters to 0 .

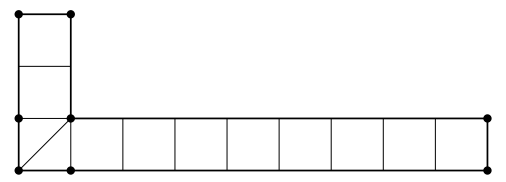

Then in the direction $(1,1)$ we see a $(1,2, n-3)$ one-cylinder surface.

5.4. L-shaped billiards. L-shaped billiards give rise to L-shaped translation surfaces by an unfolding process; any L-shaped translation (with zero twists) surface is the covering translation surface of an L-shaped billiard.

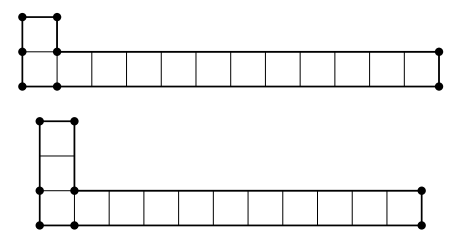

Fix some prime $n>3$, and consider the two-cylinder surfaces $S_{1}$ and $S_{2}$, both having $h_{2}=1, w_{1}=1$ and $t_{1}=t_{2}=0$, and $S_{1}$ having $h_{1}=1, w_{2}=n-1$ and $S_{2}$ having $h_{2}=2, w_{2}=n-2$. The picture on the side represents $S_{1}$ and $S_{2}$ for $n=13$. 
For each $n, S_{1}$ and $S_{2}$ belong to orbit A and B respectively, and arise from L-shaped billiards. This proves Proposition 1.3.

\section{Proof of Results about Elliptic points}

Some constructions in this section are inspired by [Ve95].

\subsection{Translations. Here we prove Proposition 4.4.}

Suppose a surface $S \in \mathcal{H}(2)$ has a nontrivial translation $f$ in its affine group. $f$ fixes the saddle and induces a permutation on outgoing horizontal separatrices. Let $\varepsilon$ be smaller than the length of the shortest saddle connection of $S$, and consider the three points at distance $\varepsilon$ from the saddle on the three separatrices in a given direction. $f$ cannot fix any of these points, otherwise it would be the identity of $S$, but it fixes the set of these points, hence it induces a cyclic permutation on them. This implies that except for the saddle, which is fixed, all $f$-orbits have size 3 . However the set of regular Weierstrass points is also fixed (since the translation $f$ is an automorphism of the underlying Riemann surface), and has size 5. This is a contradiction.

6.2. Elliptic points of order 3. Here we prove Proposition 4.5.

Suppose a surface $S$ in $\mathcal{H}(2)$ has an elliptic element of projective order three in its Veech group. Since the hyperelliptic involution has order 2, $S$ has in fact an elliptic element of order 6 in its Veech group. Conjugate by $\operatorname{SL}(2, \mathbf{R})$ to a surface that has the rotation by $\pi / 3$ (hereafter denoted by $r$ ) in its Veech group.

Considering Proposition 4.4, we denote by $r$ the corresponding affine diffeomorphism.

The set of Weierstrass points is preserved by $r$. The saddle being fixed, the remaining five Weierstrass points are setwise fixed, so at least two of them are also fixed. Consider one Weierstrass point that is fixed, call it $W$. Consider the shortest saddle connections through $W$. They come by triples making angles $\pi / 3$.

Take one such triple, consider the corresponding regular hexagon (which has these saddle connections as its diagonals).

We can take this hexagon as a building block for a polygonal fundamental domain of the surface. Consider a pair of opposite sides of this hexagon; they cannot be identified, since the rotational symmetry would imply other identifications and mean we have a torus. 
Hence, these sides and the diagonal parallel to them are three saddle connections in the same direction. So this is a completely periodic direction, and we want to see two cylinders in this direction. This would imply identifying two opposite sides, which we have excluded.

\subsection{Elliptic elements of order 2.}

6.3.1. Proof of Lemma 4.6. Here, inspired by [Ve95], we give a convenient representation for $R$-invariant Veech surfaces in $\mathcal{H}(2)$ : a fundamental octagon which is $R$-invariant. Consider a Veech surface in $\mathcal{H}(2)$ that has $R$ in its Veech group; denote also by $R$ the corresponding affine diffeomorphism.

The set of Weierstrass points is fixed by $R$ (as by any affine diffeomorphism). The saddle being fixed, at least one of the remaining 5 Weierstrass points must be fixed.

Consider such a point and the shortest saddle connections through this point. They come by orthogonal pairs. Take one such pair. Consider the square having this pair of saddle connections as diagonals. Without loss of generality, consider the sides of the square as horizontal and vertical.

This square is the central piece of our fundamental domain. Other than the corners (the saddle) and the center, there are no Weierstrass point inside this square or on its edges.

Consider the horizontal sides of our square. These sides are saddle connections so they define a completely periodic direction on the surface.

These sides are not identified, otherwise by $R$-symmetry the other two would also be and we would have a torus. So this is a two-cylinder direction and our two sides bound the short cylinder in this direction. This short cylinder lies outside the square and can be represented as a parallelogram with its "top-left" corner in the vertical strip defined by the square (i.e. with a "reasonable" twist).

By $R$-symmetry there also is such a parallelogram in the other direction. To make the picture more symmetric each parallelogram can be cut into two triangles, glued to opposite sides of the square. Thus we get a representation of the surface as an octagon with (parallel) opposite sides identified. Note that the four remaining Weierstrass points are the middle of the sides of this octagon. 
6.3.2. Proof of Proposition 4.7. Represent the surface as above: an octagon made of a square and four triangles glued to its sides. All vertices lie on integer points.

Let $A B C$ be one of the triangles, labeled clockwise so that $A C$ is a side of the square.

Let $(p, q)$ be the coordinates of $\overrightarrow{A C}$ and $(r, s)$ those of $\overrightarrow{A B}$. The area of the surface is then $p^{2}+q^{2}+2(p s-q r)$.

If $n$ is prime then $p$ and $q$ have to be relatively prime, and of different parity. Then $p^{2}+q^{2} \equiv 1[4]$. The center of the square lies at the center of a square of the tiling.

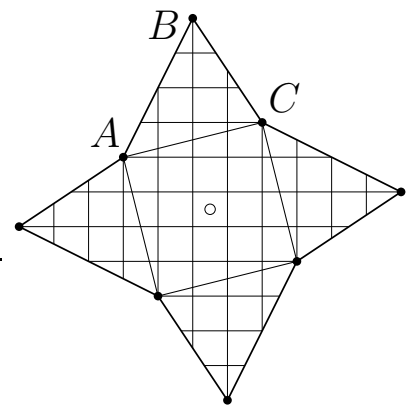
The condition for two Weierstrass points to lie on integer points is for $(p s-r q)$ to be even.

We conclude by observing that $n$ is 1 (resp. 3) modulo 4 when $(p s-r q)$ is even (resp. odd).

\section{Proof of COUntings}

Here we establish the countings and estimates of Proposition 4.8.

7.1. One-cylinder cusps and surfaces. For prime $n>3$, onecylinder $n$-square-tiled cusps in $\mathcal{H}(2)$ are in $1-1$ correspondence with cyclically ordered 3-partitions of $n$.

Ordered 3-partitions $(a, b, c)$ of $n$ are in 1-1 correspondence with pairs of distinct integers $\{\alpha, \beta\}$ in $\{1, \ldots, n-1\}$ : assuming $\alpha<\beta$, the correspondence is given by $a=\alpha, a+b=\beta, a+b+c=n$. So there are $C_{n-1}^{2}$ ordered 3-partitions of $n$. Ordered 3-partitions of $n$ being in 3-1 correspondence with cyclically ordered 3-partitions, there are $\frac{1}{3} C_{n-1}^{2}=\frac{(n-1)(n-2)}{6}$ cyclically ordered 3-partitions of $n$.

Thus there are $\frac{(n-1)(n-2)}{6}$ one-cylinder cusps of $n$-square-tiled translation surfaces in $\mathcal{H}(2)$.

Those in orbit A are those with 3 odd parts $2 a-1,2 b-1,2 c-1$; these are in 1-1 correspondence with cyclically ordered partitions $a$, $b, c$, of $\frac{n+3}{2}$. Their number is hence $\frac{1}{6}\left(\frac{n+3}{2}-1\right)\left(\frac{n+3}{2}-2\right)=\frac{(n+1)(n-1)}{24}$.

The remaining ones are in orbit $B$, their count is hence the difference, $\frac{(n-1)(n-3)}{8}$. 
All one-cylinder cusps discussed here have width $n$ ( $n$ possible values of the twist parameter), so the counts of one-cylinder surfaces are $n$ times the corresponding cusp counts.

7.2. Two-cylinder surfaces. The total number of two-cylinder $n$ square-tiled surfaces ( $n$ prime) is

$$
S(n)=\sum_{a, b, k, \ell} k \ell
$$

where the sum is over $a, b, k, \ell \in \mathbf{N}^{*}$ such that $k<\ell$ and $a k+b \ell=n$.

This follows from the parametrization in $\S 3.3 .2$; the letters $a, b, k$, $\ell$ used here correspond to the parameters $h_{1}, h_{2}, w_{1}, w_{2}$ there, and the summand is the number of possible values of the twist parameters, given the heights and widths of the two cylinders.

We want the asymptotic for this quantity as $n$ tends to infinity, $n$ prime. In order to find this, we consider the sum as a double sum: the sum over $a$ and $b$ of the sum over $k$ and $\ell$.

Write $S(n)=\sum_{a, b} S_{a, b}(n)$, where $S_{a, b}(n)=\sum_{k, \ell} k \ell$.

We study the inner sum by analogy with a payment problem: how many ways are there to pay $n$ units with coins worth $a$ and $b$ units?

This problem is classically solved by the use of generating series: denote the number of ways to pay by $s_{a, b}(n)$; then

$$
s_{a, b}(n)=\operatorname{Card}\left\{(k, \ell) \in \mathbf{N}^{2}: a k+b \ell=n\right\}=\sum_{k, \ell \in \mathbf{N}: a k+b \ell=n} 1 .
$$

Now notice that $\sum_{k=0}^{\infty} z^{a k} \sum_{\ell=0}^{\infty} z^{b \ell}=\sum_{n=0}^{\infty} s_{a, b}(n) z^{n}$, and deduce that the number looked for is the $n$-th coefficient of the power series expansion of the function $\frac{1}{1-z^{a}} \frac{1}{1-z^{b}}$.

We turn back to our real problem, $S_{a, b}(n)=\sum_{k, \ell \in \mathbf{N}^{*}: a k+b \ell=n, k<\ell} k \ell$.

We want to show that $S(n) \sim c n^{3}$ for prime $n$. For this we will use the dominated convergence theorem: we show that $S_{a, b}(n) / n^{3}$ has a limit $c_{a, b}$ when $n$ tends to infinity with $a$ and $b$ fixed, and that $S_{a, b}(n) / n^{3}$ is bounded by some $g_{a, b}$ such that $\sum_{a, b} g_{a, b}<\infty$, to conclude that $S(n) / n^{3}=\sum_{a, b} S_{a, b}(n) / n^{3}$ tends to $c=\sum_{a, b} c_{a, b}$, which means $S(n) \sim c n^{3}$.

The dominated convergence is proved as follows. 
Write $S_{a, b}(n)=\sum_{k, h \in \mathbf{N}^{*}:(a+b) k+b h=n} k(k+h)$ by introducing $h=$ $\ell-k$. Then split the sum into $\sum k^{2}$ and $\sum k h$. Write

$$
\begin{aligned}
& S_{a, b}^{\prime}(n)=\sum_{k, h \in \mathbf{N}^{*},} k^{2} / n^{3} \leqslant \sum_{k \in \mathbf{N}^{*}, h \in \mathbf{Q},(a+b) k+b h=n} k^{2} / n^{3} \\
& S_{a, b}^{\prime \prime}(n)=\sum_{k, h \in \mathbf{N}^{*},(a+b) k+b h=n} k h / n^{3} \leqslant \sum_{k \in \mathbf{N}^{*}, h \in \mathbf{Q},(a+b) k+b h=n} k h / n^{3}
\end{aligned}
$$

(in the sums on the right-hand side, $h$ has been allowed to be a rational instead of an integer.) Hence

$$
\begin{gathered}
S_{a, b}^{\prime}(n) \leqslant \frac{1}{(a+b)^{3}}\left[\frac{a+b}{n} \sum_{k=1}^{\lfloor n /(a+b)\rfloor}\left(\frac{a+b}{n} k\right)^{2}\right] \\
S_{a, b}^{\prime \prime}(n) \leqslant \frac{1}{(a+b)^{2} b}\left[\frac{a+b}{n} \sum_{k=0}^{n /(a+b)}\left(\frac{a+b}{n} k\right)\left(1-\frac{a+b}{n} k\right)\right]
\end{gathered}
$$

The expressions in brackets, Riemann sum approximations to the integrals $\int_{0}^{1} x^{2} \mathrm{~d} x$ and $\int_{0}^{1} x(1-x) \mathrm{d} x$, are uniformly bounded by 1 .

Now notice that $\sum_{a, b} \frac{1}{(a+b)^{3}}$ and $\sum_{a, b} \frac{1}{(a+b)^{2} b}$ are convergent. This ends the dominated convergence argument.

We can now investigate the limit. For ease of calculation, we drop the condition $k<\ell$. We take care of it by writing $\sum_{k, \ell}=$ $2 \sum_{k<\ell}+\sum_{k=\ell}$. For prime $n, k=\ell$ implies that they are both equal to 1 . The sum for $k=\ell$ is hence equal to $n-1$, and we will not need to take it into account since the whole sum will grow as $n^{3}$.

Denote by $\tilde{S}(n, a, b)$ the sum over all $k$ and $\ell$.

Notice that $\sum_{k=0}^{\infty} k z^{a k} \sum_{\ell=0}^{\infty} \ell z^{b \ell}=\sum_{n=0}^{\infty} \tilde{S}(n, a, b) z^{n}$.

$\tilde{S}(n, a, b)$ is therefore the $n$-th coefficient of the power series expansion of the function $f_{a, b}=\frac{z^{a}}{\left(1-z^{a}\right)^{2}} \frac{z^{b}}{\left(1-z^{b}\right)^{2}}$.

To determine this coefficient, decompose $f_{a, b}$ into partial fractions. This function has poles at $a$-th and $b$-th roots of 1 . Since $n$ is prime, we are only interested in relatively prime $a$ and $b$, for which the only common root of 1 is 1 itself, which is hence a 4 -th order pole of $f_{a, b}$, while other poles have order 2 .

The $n$-th coefficient of the power series expansion of $f_{a, b}$ is a polynomial of degree 3 in $n$, whose leading term is $c_{a, b} \frac{n^{3}}{6}$, where $c_{a, b}$ is the 
coefficient of $\frac{1}{(1-z)^{4}}$ in the decomposition of $f_{a, b}$ into partial fractions. This coefficient is computed to be $\frac{1}{a^{2} b^{2}}$.

We want the sum over relatively prime $a$ and $b$. We relate it to the sum over all $a$ and $b$ by sorting the latter according to $d=a \wedge b$.

$$
\sum_{a, b} \frac{1}{a^{2} b^{2}}=\sum_{d} \sum_{a, b, a \wedge b=d} \frac{1}{a^{2} b^{2}}=\sum_{d} \frac{1}{d^{4}} \sum_{a, b, a \wedge b=1} \frac{1}{a^{2} b^{2}} .
$$

By observing that $\sum_{a, b} \frac{1}{a^{2} b^{2}}=\left(\sum_{a} \frac{1}{a^{2}}\right)^{2}=\zeta(2)^{2}=\frac{\pi^{4}}{36}$ and that $\sum_{d} \frac{1}{d^{4}}=\zeta(4)=\frac{\pi^{4}}{90}$ we get that the sum $\sum_{a, b, a \wedge b=1} \frac{1}{a^{2} b^{2}}$ is equal to $5 / 2$. Divide by 2 to get back to $k<\ell$, and find that $S(n) \sim \frac{5}{4} \frac{n^{3}}{6}$.

7.3. Two-cylinder surfaces by orbit. Two-cylinder surfaces for which both heights are odd are in orbit A; those for which both widths are odd are in orbit B; half of the remaining ones are in orbit $\mathrm{A}$, and half in $\mathrm{B}$; the factor one half comes from the conditions on the twists. (See the table in $\S 5.1$.)

First compute the asymptotic for odd heights. Write

$$
S^{\mathrm{oh}}(n)=\sum_{\substack{a, b, k, \ell \\ a k+b \ell=n \\ a, b \text { odd } \\ a \wedge b=1 \\ k<\ell}} k \ell .
$$

Then $S^{\text {oh }}(n) \sim \frac{1}{2} \widetilde{S}^{\text {oh }}(n)$ where $\widetilde{S}^{\text {oh }}(n)$ is the same sum without the condition $k<\ell$. The dominated convergence works as previously.

For odd $a$ and $b$ such that $a \wedge b=1$,

$$
\widetilde{S}_{a, b}^{\mathrm{oh}}(n)=\sum_{\substack{k, \ell \\ a k+b \ell=n}} k \ell \sim \frac{1}{a^{2} b^{2}} \cdot \frac{n^{3}}{6} .
$$

We need to sum over relatively prime odd $a$ and $b$. Using the same trick as previously, write

$$
\sum_{a, b \text { odd }} \frac{1}{a^{2} b^{2}}=\sum_{d \text { odd }} \sum_{\substack{a, b \text { odd } \\ a \wedge b=d}} \frac{1}{a^{2} b^{2}}=\sum_{d \text { odd }} \frac{1}{d^{4}} \sum_{\substack{a, b \text { odd } \\ a \wedge b=1}} \frac{1}{a^{2} b^{2}} .
$$


Now

$$
\sum_{a, b \text { odd }} \frac{1}{a^{2} b^{2}}=\left(\sum_{a \text { odd }} \frac{1}{a^{2}}\right)^{2}=\left(\left(1-1 / 2^{2}\right) \zeta(2)\right)^{2}=\frac{9}{16} \cdot \frac{\pi^{4}}{36}
$$

and

$$
\sum_{d \text { odd }} \frac{1}{d^{4}}=\left(1-1 / 2^{4}\right) \zeta(4)=\frac{15}{16} \cdot \frac{\pi^{4}}{90}
$$

SO

$$
\sum_{\substack{a, b \text { odd } \\ a \wedge b=1}} \frac{1}{a^{2} b^{2}}=3 / 2
$$

We deduce that $S^{\mathrm{oh}}(n) \sim \frac{3}{4} \frac{n^{3}}{6}$ (the condition $k<\ell$ is responsible for a factor $1 / 2$ ).

Similarly compute the asymptotic for odd widths. Write

$$
S^{\text {ow }}(n)=\sum_{\substack{a, b, k, \ell \\ a k+b \ell=n \\ k, \ell \text { odd } \\ a \wedge b=1 \\ k<\ell}} k \ell .
$$

For fixed $a$ and $b$ with $a \wedge b=1$, put

$$
\widetilde{S}_{a, b}^{\text {ow }}(n)=\sum_{\substack{k, \ell \text { odd } \\ a k+b \ell=n}} k \ell
$$

Notice that $\sum_{k \text { odd }} k z^{a k} \sum_{\ell \text { odd }} \ell z^{b \ell}=\sum \widetilde{S}_{a, b}^{\text {ow }}(n) z^{n}$.

Because $\sum k z^{k}=\frac{z}{(1-z)^{2}}, \sum 2 k z^{2 k}=\frac{2 z^{2}}{\left(1-z^{2}\right)^{2}}$, and the difference is $\sum(2 k+1) z^{2 k+1}=\frac{z\left(1+z^{2}\right)}{\left(1-z^{2}\right)^{2}}$.

$\widetilde{S}_{a, b}^{\text {ow }}(n)$ is now the $n$-th coefficient of the power series expansion of $\frac{z^{a}\left(1+z^{2 a}\right)}{\left(1-z^{2 a}\right)^{2}} \cdot \frac{z^{b}\left(1+z^{2 b}\right)}{\left(1-z^{2 b}\right)^{2}}$. When $a \wedge b=1$, this rational function has two order 4 poles at 1 and -1 and its other poles have order 2; the coefficients of $\frac{1}{(1-z)^{4}}$ and $\frac{1}{(1+z)^{4}}$ in its decomposition into partial fractions are respectively $\frac{1}{4 a^{2} b^{2}}$ and $\frac{(-1)^{a+b}}{4 a^{2} b^{2}}$.

Because $n$ is odd, and $k$ and $\ell$ are odd, $a$ and $b$ have to have different parities, so $a+b$ is odd. So $\frac{1}{4 a^{2} b^{2}}+\frac{(-1)^{a+b}(-1)^{n}}{4 a^{2} b^{2}}=\frac{1}{2 a^{2} b^{2}}$. 
Now

$$
\sum_{\substack{a \wedge b=1 \\ a \neq b[2]}} \frac{1}{a^{2} b^{2}}=\sum_{a \wedge b=1} \frac{1}{a^{2} b^{2}}-\sum_{\substack{a \wedge b=1 \\ a, b \text { odd }}} \frac{1}{a^{2} b^{2}}=5 / 2-3 / 2=1 .
$$

The condition $k<\ell$ brings a factor $1 / 2$, thus we get $S^{\text {ow }}(n) \sim$ $(1 / 4)\left(n^{3} / 6\right)$.

The remaining surfaces are those for which heights as well as widths of the cylinders have mixed parities. The asymptotic for this "evenodd" part is computed as the difference between the total sum and the odd-widths and odd-heights sums.

Write $S(n)=S^{\text {oh }}(n)+S^{\text {ow }}(n)+S^{\mathrm{eo}}(n)$. We already know that $S(n) \sim \frac{5}{4} \cdot \frac{n^{3}}{6}, S^{\mathrm{oh}}(n) \sim \frac{3}{4} \cdot \frac{n^{3}}{6}$, and $S^{\text {ow }}(n) \sim \frac{1}{4} \cdot \frac{n^{3}}{6}$. So the even-odd part has asymptotics $S^{\mathrm{eo}}(n) \sim \frac{1}{4} \cdot \frac{n^{3}}{6}$.

Putting pieces together, the number of $n$-square-tiled two-cylinder surfaces of type A, $n$ prime, is equivalent to $(3 / 4+1 / 8)\left(n^{3} / 6\right)=$ $(7 / 8)\left(n^{3} / 6\right)$. For type $\mathrm{B}$, we get $(1 / 4+1 / 8)\left(n^{3} / 6\right)=(3 / 8)\left(n^{3} / 6\right)$.

7.4. Two-cylinder cusps. For $n$ prime, the number of two-cylinder cusps (in both orbits) is given by

$$
S(n)=\sum_{\substack{a, b, k, \ell \in \mathbf{N}^{*} \\ a k+b \ell=n \\ k<\ell}}(a \wedge k)(b \wedge \ell) .
$$

(see counting of two-cylinder surfaces in $\S 7.2$ and discussion of cusps in $\S 3.4$.)

Remark. For nonprime $n$, the number of two-cyl cusps is less than $S(n)$ defined as above, so the bound found here is still valid.

$S(n)$ is less than

$$
\widetilde{S}(n)=\sum_{\substack{a, b, k, \ell \in \mathbf{N}^{*} \\ a k+b \ell=n}}(a \wedge k)(b \wedge \ell) .
$$

where the condition $k<\ell$ is dropped.

We will show that for any $\varepsilon>0, \widetilde{S}(n) \ll_{n \rightarrow \infty} n^{3 / 2+\varepsilon}$.

This will imply that the number of two-cylinder cusps of $n$-squaretiled surfaces is sub-quadratic, thus negligible before the (quadratic) number of one-cylinder cusps in each orbit. 


$$
\widetilde{S}(n)=\sum_{\substack{A, B, u, v \in \mathbf{N}^{*} \\ A u^{2}+B v^{2}=n}} u v f(A) f(B), \quad \text { where } f(m)=\sum_{\substack{r s=m \\ r \wedge s=1}} 1 .
$$

Note that $f(m) \leqslant \mathrm{d}(m) \ll m^{\varepsilon}$, where $\mathrm{d}(m)$ is the number of divisors of $m$. The factors $f(A) f(B)$ therefore contribute less than an $n^{\varepsilon}$.

$$
\sum_{\substack{A, B, u, v \in \mathbf{N}^{*} \\ A u^{2}+B v^{2}=n}} u v=\sum_{u} u \sum_{A \leqslant n / u^{2}}\left(\sum_{v^{2} \mid n-A u^{2}} v\right) .
$$

The sum in parentheses has less than $\mathrm{d}\left(n-A u^{2}\right)$ summands, each of which is bounded by $\sqrt{n-A u^{2}}$, so

$$
\widetilde{S}(n) \ll n^{1 / 2+2 \varepsilon} \sum_{u} n / u \ll n^{3 / 2+3 \varepsilon} .
$$

We thank Joël Rivat for contributing this estimate [Ri].

7.5. Elliptic points. The discussion in $\S 6.3 .2$ implies that their number is less than the number of integer-coordinate vectors in a quarter of a circle of radius $\sqrt{n}$, so it is $O(n)$.

\section{Strong Numerical EVIDENCE}

Martin Schmoll pointed out to us that the number of primitive $n$-square-tiled surfaces in $\mathcal{H}(2)$ is given in [EsMaSc] to be

$$
\frac{3}{8}(n-2) n^{2} \prod_{p \mid n}\left(1-\frac{1}{p^{2}}\right)
$$

By [Mc2], for even $n$ all these surfaces are in the same orbit, and for odd $n \geqslant 5$ they fall into two orbits. So Eskin, Masur and Schmoll's formula gives the cardinality of the single orbit for even $n$, and the sum of the cardinalities of the two orbits for odd $n$.

Conjecture 8.1. For odd n, the cardinalities of the orbits are given by the following functions:

orbit $A: \frac{3}{16}(n-1) n^{2} \prod_{p \mid n}\left(1-\frac{1}{p^{2}}\right)$,

orbit $B: \frac{3}{16}(n-3) n^{2} \prod_{p \mid n}\left(1-\frac{1}{p^{2}}\right)$. 
These formulae give degree 3 polynomials when restricted to prime $n$, for which Theorem 1.2 gives the leading term. These polynomials are expressed in the table below.

\begin{tabular}{lccc}
\hline & one-cylinder & two-cylinder & all \\
\hline $\mathrm{A}$ & $\frac{1}{24}\left(n^{3}-n\right)$ & $\frac{1}{48}\left(7 n^{3}-9 n^{2}-7 n+9\right)$ & $\frac{3}{16}\left(n^{3}-n^{2}-n+1\right)$ \\
\hline $\mathrm{B}$ & $\frac{1}{8}\left(n^{3}-4 n^{2}+3 n\right)$ & $\frac{1}{16}\left(n^{3}-n^{2}-9 n+9\right)$ & $\frac{3}{16}\left(n^{3}-3 n^{2}-n+3\right)$ \\
\hline all & $\frac{1}{6}\left(n^{3}+3 n^{2}+2 n\right)$ & $\frac{1}{24}\left(5 n^{3}-6 n^{2}-17 n+18\right)$ & $\frac{3}{8}\left(n^{3}-2 n^{2}-n+2\right)$ \\
\hline
\end{tabular}

On the other hand, the counting functions for two-cylinder cusps are not polynomials.

Conjecture 8.2. For prime n, the number of elliptic points is $\left\lfloor\frac{n+1}{4}\right\rfloor$.

This conjecture is valid for the first thousand odd primes.

ApPENDIX A. $n=3$ AND $n=5$

$n=3$. For $n=3$, we have the following three surfaces.

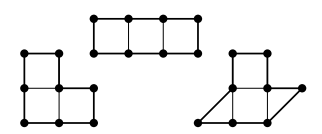

If we call $S_{1}$ the one-cylinder surface, and $S_{2}$ and $S_{3}$ the two-cylinder surfaces, the generators of $\mathrm{SL}(2, \mathbf{Z})$ act as follows: $U S_{1}=S_{1}, U S_{2}=S_{3}$, $U S_{3}=S_{2}, R S_{1}=S_{3}, R S_{2}=S_{2}, R S_{3}=S_{1}$. So there is only one orbit, containing $d=3$ surfaces, the number of cusps is $c=2$, the number of elliptic points ( $R$-invariant surfaces) is $e=1$, so the genus is $g=0$ by the Gauss-Bonnet formula.

$n=5$. For $n=5$, we have 27 surfaces forming 8 cusps, a representative of which appears on the following picture.
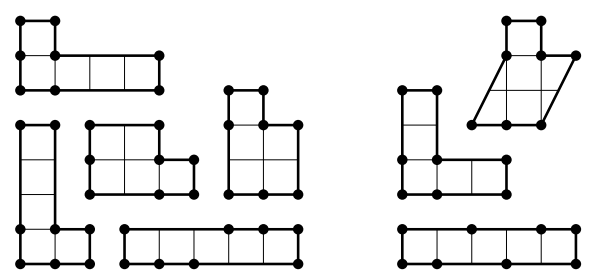

Computing the $\operatorname{SL}(2, \mathbf{Z})$ action shows that they fall into two orbits, orbit A being made of the surfaces on the left and orbit B of those on the right.

The data for orbit A is $d=18$ surfaces, $c=5$ cusps, $e=0$ elliptic point, so the genus is $g=0$ by the Gauss-Bonnet formula. 
The data for orbit $\mathrm{B}$ is $d=9$ surfaces, $c=3$ cusps, $e=1$ elliptic point, so the genus is $g=0$ by the Gauss-Bonnet formula.

By inspection of the congruence subgroups of genus 0 of $\operatorname{SL}(2, \mathbf{Z})$ (see for example $[\mathrm{CuPa}]$ ), the stabilizers of orbits $\mathrm{A}$ and $\mathrm{B}$ are noncongruence subgroups of $\operatorname{SL}(2, \mathbf{Z})$.

\section{ApPendix B. HyperelliptiC COMPONENTS OF OTHER STRATA}

For all hyperelliptic square-tiled surfaces, one can count the number of Weierstrass points with integer coordinates. This provides an invariant for the action of $\mathrm{SL}(2, \mathbf{Z})$ on square-tiled surfaces in all hyperelliptic components of strata of moduli spaces of abelian differentials.

The strata with hyperelliptic components are $\mathcal{H}(2 g-2)$ and $\mathcal{H}(g-$ $1, g-1)$, for $g>1$.

Proposition B.1. In $\mathcal{H}(2 g-2)^{\text {hyp }}$ and $\mathcal{H}(g-1, g-1)^{\text {hyp }}$, for large enough odd $n$ there are at least $g$ orbits containing one-cylinder surfaces.

This is proved by the following reasoning.

Completely periodic surfaces in $\mathcal{H}(2 g-2)$ or $\mathcal{H}(g-1, g-1)$, for $g>1$, have respectively $2 g-1$ and $2 g$ saddle connections.

For one-cylinder primitive surfaces (necessarily of height 1), the lengths of the saddle connections add up to $n$, and the Weierstrass points are two points on the circle at half-height of this cylinder (these do not have integer coordinates), the saddle in the $\mathcal{H}(2 g-2)^{\text {hyp }}$ case, and the midpoints of the saddle connections that bound the cylinder (these have integer coordinates for exactly those saddle connections of even length).

If $n$ is odd, the sum of the lengths is odd. So the number of oddlength saddle connections has to be odd, and is between 1 and $2 g-1$. There are $g$ possibilities for that. Since the value of the invariant is the number of even-length saddle connections, it can take $g$ different values.

\section{Appendix C. The theorem of Gutkin and Judge}

Theorem (Gutkin-Judge). $(S, \omega)$ has an arithmetic Veech group if and only if $(S, \omega)$ is parallelogram-tiled. 
Up to conjugating by an element of $\operatorname{SL}(2, \mathbf{R})$, it suffices to show:

Theorem. $(S, \omega)$ is a square-tiled surface if and only if $V(S, \omega)$ is commensurable to $\mathrm{SL}(2, \mathbf{Z})$.

(i.e. these two groups share a common subgroup of finite index in each.)

Remark. In this theorem, the size of the square tiles is not assumed to be 1 . One can always act by a homothety to make this true, and we will suppose that in the proof of the direct way of this theorem.

C.1. A square-tiled surface has an arithmetic Veech group. Consider a square-tiled surface $(S, \omega)$, and its lattice of periods $\Lambda(\omega)$. By Lemma 2.3, $V(S, \omega)<V\left(\mathbf{R}^{2} / \Lambda(\omega), \mathrm{d} z\right)$.

Case 1. Let us first assume that $\Lambda(\omega)=\mathbf{Z}^{2}$, i.e. $(S, \omega)$ is a primitive square-tiled surface.

Lemma 2.4 implies that $\mathrm{SL}(2, \mathbf{Z})$ acts on the set $E$ of square-tiled surfaces contained in its $\operatorname{SL}(2, \mathbf{R})$-orbit. The set $E$ is finite and the stabilizer of this action is $V(S, \omega)$. The class formula then implies that $V(S, \omega)$ has finite index in $\operatorname{SL}(2, \mathbf{Z})$.

Case 2. Suppose that $\Lambda(\omega)$ is a strict sublattice of $\mathbf{Z}^{2}$. Consider $P_{1}, \ldots, P_{k}$ the preimages of the origin on $S$. Denote by Aff $_{P_{1}, \ldots, P_{k}}$ the stabilizer of the set of these points in the affine group of $(S, \omega)$, and $V\left(P_{1}, \ldots, P_{k}\right)$ the associated Veech group. The translation surface $\left(S, \omega,\left\{P_{1}, \ldots, P_{k}\right\}\right)$ where $\left\{P_{1}, \ldots, P_{k}\right\}$ are artificially marked is a primitive square-tiled surface. From Case 1 above, its Veech group $V\left(P_{1}, \ldots, P_{k}\right)$ is therefore a lattice contained in the discrete group $V(S, \omega)$, hence of finite index in this group.

Thus $V\left(P_{1}, \ldots, P_{k}\right)$ is a finite-index subgroup in both $V(S, \omega)$ and $\mathrm{SL}(2, \mathbf{Z})$.

C.2. A surface with an arithmetic Veech group is squaretiled. This part is inspired by ideas of Thurston [Th] and Veech [Ve89, §9], and appeared in [Hu, appendix B].

Let $S$ be a translation surface with an arithmetic Veech group $\Gamma$.

If $\Gamma$ is commensurable to $\operatorname{SL}(2, \mathbf{Z})$ only in the wide sense, we move to the case of strict commensurability. This conjugacy on Veech groups is obtained by $\mathrm{SL}(2, \mathbf{R})$ action on surfaces.

We prove the following propositions. 
Proposition C.1. A group $\Gamma$ commensurable with $\mathrm{SL}(2, \mathbf{Z})$ contains two elements of the form $\left(\begin{array}{ll}1 & m \\ 0 & 1\end{array}\right)$ and $\left(\begin{array}{ll}1 & 0 \\ n & 1\end{array}\right)$ for some $m, n \in \mathbf{N}^{*}$.

Proposition C.2. If the Veech group $\Gamma$ of a translation surface $S$ contains two elements of the form $\left(\begin{array}{ll}1 & m \\ 0 & 1\end{array}\right)$ and $\left(\begin{array}{ll}1 & 0 \\ n & 1\end{array}\right)$ for some $m, n \in$ $\mathbf{N}^{*}$, then $S$ is square-tiled.

Proposition C.1 follows from the following lemma.

Lemma C.3. If $H \leq G$ is a finite-index subgroup then every $g \in G$ of infinite order has a power in $H$.

Proof of the lemma. If $H$ has finite index there is a partition of $G$ into a finite number of classes modulo $H$. The powers of $g$, in countable number, are distributed in these classes, so there exist distinct integers $i$ and $j$ such that $g^{i}$ and $g^{j}$ are in the same class, and then $g^{j-i} \in H$.

Apply this lemma to $G=\operatorname{SL}(2, \mathbf{Z})$ and $H$ the common subgroup to $G$ and $\Gamma$, of finite index in both $G$ and $\Gamma$, and $g=\left(\begin{array}{ll}1 & 1 \\ 0 & 1\end{array}\right)$ or $g=\left(\begin{array}{ll}1 & 0 \\ 1 & 1\end{array}\right)$.

We now prove Proposition C.2.

Since $\left(\begin{array}{cc}1 & m \\ 0 & 1\end{array}\right) \in \Gamma$, the horizontal direction is parabolic, so $S$ decomposes into horizontal cylinders $C_{i}^{\mathrm{h}}$ of rational moduli. Replacing $\left(\begin{array}{cc}1 & m \\ 0 & 1\end{array}\right)$ with one of its powers if necessary, suppose it fixes the boundaries of these cylinders. This means their moduli are multiples of $1 / m$. Calling $w_{i}^{\mathrm{h}}, h_{i}^{\mathrm{h}}$ the widths and heights of these cylinders, we have relations $h_{i}^{\mathrm{h}} / w_{i}^{\mathrm{h}}=k_{i} / m$ for some integers $k_{i}$.

By a similar argument, since $\left(\begin{array}{ll}1 & 0 \\ n & 1\end{array}\right) \in \Gamma$, the vertical direction is also parabolic, and $S$ decomposes into vertical cylinders $C_{j}^{\mathrm{v}}$ of rational moduli $h_{j}^{\mathrm{v}} / w_{j}^{\mathrm{v}}=k_{j}^{\prime} / n$ for some integers $k_{j}^{\prime}$.

Combining these two decompositions yields a decomposition of $S$ into rectangles of dimensions $h_{j}^{\mathrm{v}} \times h_{i}^{\mathrm{h}}$ (these rectangles are the connected components of the intersections of the horizontal and vertical cylinders). Here we keep on with the convention of $\S 2.3$ about heights and widths of cylinders.

What we want to show is that these rectangles have rational dimensions (up to a common real scaling factor), in order to prove that $S$ is a covering of a square torus; indeed, if the rectangles are such, then they can be divided into equal squares, so we obtain a covering of a square torus. Since singular points of $S$ lie on the edges both of 
horizontal and of vertical cylinders, they are at corners of rectangles and hence of squares of the tiling, so that the covering is ramified over only one point.

Because the cylinders in the decompositions above are made up of these rectangles, we have $w_{i}^{\mathrm{h}}=\sum m_{i j} h_{j}^{\mathrm{v}}$ and $w_{j}^{\mathrm{v}}=\sum n_{j i} h_{i}^{\mathrm{h}}$, where $m_{i j}, n_{j i} \in \mathbf{N}$.

Combining equations, $m h_{i}^{h}=\sum k_{i} m_{i j} h_{j}^{v}$ and $n h_{j}^{v}=\sum k_{j}^{\prime} n_{j i} h_{i}^{h}$.

Then, setting $X^{\mathrm{h}}=\left(h_{i}^{\mathrm{h}}\right), X^{\mathrm{v}}=\left(h_{j}^{\mathrm{v}}\right), M=\left(k_{i} m_{i j}\right)_{i j}, N=\left(k_{j}^{\prime} n_{j i}\right)_{j i}$, we have $m X^{\mathrm{h}}=M X^{\mathrm{v}}$ and $n X^{\mathrm{v}}=N X^{\mathrm{h}}$, so that $M N X^{\mathrm{h}}=m n X^{\mathrm{h}}$ and $N M X^{\mathrm{v}}=n m X^{\mathrm{v}}$.

$M, N$ and their products are matrices with nonnegative integer coefficients. In view of applying the Perron-Frobenius theorem, we show that MN and NM have powers with all coefficients positive.
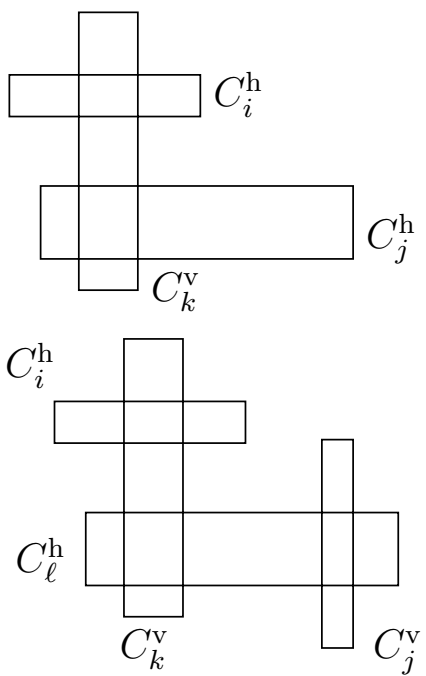

This results from the connectedness of $S$ and the following observation: $M_{i j} \neq$ 0 if and only if $C_{i}^{\mathrm{h}}$ and $C_{j}^{\mathrm{v}}$ intersect; $(M N)_{i j} \neq 0$ if and only if there exists a cylinder $C_{k}^{\mathrm{v}}$ which intersects both $C_{i}^{\mathrm{h}}$ and $C_{j}^{\mathrm{h}}$, as in the picture; more generally the element $i, j$ of a product of alternately $M$ and $N$ matrices is nonzero if and only if there exists a corresponding sequence of alternately horizontal and vertical cylinders such that two successive cylinders intersect. So $M N$ and $N M$ do have powers with all coefficients positive.

$X^{\mathrm{h}}\left(\operatorname{resp} . X^{\mathrm{v}}\right)$ is an eigenvector for the eigenvalue $n m$ of the square matrix $M N$ (resp. $N M$ ). By the PerronFrobenius theorem, there exists a unique eigenvector associated with the real positive eigenvalue $\mathrm{nm}$ for the matrix $N M$ (resp. $M N$ ). Since both matrices have rational coefficients and the eigenvalue is rational, there exist eigenvectors with rational coefficients. Up to scaling, they are unique by the Perron-Frobenius theorem. This allows to conclude that $X^{\mathrm{h}}$ is a multiple of a vector with rational coordinates. From the equation $n X^{\mathrm{v}}=N X^{\mathrm{h}}$, we then conclude that the rectangles have rational moduli and can be tiled by identical squares. This completes the proof of the theorem. 
C.3. A corollary. The following result of $[\mathrm{GuHuSc}]$ arises as a corollary of $\S$ C.1 and Proposition C.2.

Corollary C.4. If a subgroup $\Gamma<\mathrm{SL}(2, \mathbf{Z})$ contains two elements $\left(\begin{array}{ll}1 & m \\ 0 & 1\end{array}\right)$ and $\left(\begin{array}{ll}1 & 0 \\ n & 1\end{array}\right)$ and has infinite index in $\mathrm{SL}(2, \mathbf{Z})$, then $\Gamma$ cannot be realized as the Veech group of a translation surface.

\section{REFERENCES}

[Ca] K. Calta. Veech surfaces and complete periodicity in genus 2. Preprint. arXiv:math.DS/0205163

[CuPa] C. J. Cummins, S. Pauli. Congruence subgroups of PSL(2,Z) of genus less than or equal to 24. Experimental mathematics 12:2 (2003), 243-255. http://www.math.tu-berlin.de/ pauli/congruence/

[EsMaSc] A. Eskin, H. Masur, M. Schmoll. Billiards in rectangles with barriers. Duke Math. J. 118:3 (2003) 427-463.

[EsOk] A. Eskin, A. Okounkov. Asymptotics of numbers of branched coverings of a torus and volumes of moduli spaces of holomorphic differentials. Invent. Math. 145:1 (2001), 59-103.

[Gu] E. Gutkin. Billiards on almost integrable polyhedral surfaces. Ergodic Theory Dynam. Systems 4:4 (1984) 569-584.

[GuHuSc] E. Gutkin, P. Hubert, T. Schmidt. Affine diffeomorphisms of translation surfaces: periodic points, fuchsian groups, and arithmeticity. To appear in Ann. Sci. École Norm. Sup. (4).

[GuJu1] E. Gutkin, C. Judge. The geometry and arithmetic of translation surfaces with applications to polygonal billiards. Math. Res. Lett. 3:3 (1996), 391-403.

[GuJu2] E. Gutkin, C. Judge. Affine mappings of translation surfaces: geometry and arithmetic. Duke Math. J. 103:2 (2000), 191-213.

[Hu] P. Hubert. Étude géométrique et combinatoire de systèmes dynamiques d'entropie nulle. Habilitation à diriger des recherches. 2002.

[HL] P. Hubert, S. Lelièvre. Noncongruence subgroups in $\mathcal{H}(2)$. To appear in Internat. Math. Res. Notices.

[HuSc00] P. Hubert, T. Schmidt. Veech groups and polygonal coverings. J. Geom. Physics 35:1 (2000), 75-91.

[HuSc01] P. Hubert, T. Schmidt. Invariants of translation surfaces. Ann. Inst. Fourier (Grenoble) 51:2 (2001), 461-495.

[Ka] E. Kani. The number of genus 2 covers of an elliptic curve. Preprint (2003).

[KeMaSm] S. Kerckhoff, H. Masur, J. Smillie. Ergodicity of billiard flows and quadratic differentials. Ann. of Math. (2) 124:2 (1986), 293-311.

[KeSm] R. Kenyon, J. Smillie. Billiards on rational-angled triangles. Comment. Math. Helv. 75:1 (2000), 65-108. 
[KoZo] M. Kontsevich, A. Zorich. Connected components of the moduli space of holomorphic differentials with prescribed singularities. Invent. Math. 153:3 (2003), 631-678.

[Ma] H. Masur. Interval exchange transformations and measured foliations. Ann. of Math. (2) 115:1 (1982), 169-200.

[Mc] C. T. McMullen. Billiards and Teichmüller curves on Hilbert modular surfaces. J. Amer. Math. Soc. 16:4 (2003), 857-885.

[Mc2] C. T. McMullen. Teichmüller curves in genus two: discriminant and spin. Preprint (2004).

[Mö] M. Möller. Teichmüller curves, Galois actions and $\widehat{G T}$-relations. Preprint (2003). arXiv:math.AG/0311308

[Ri] J. Rivat. Private communication.

[Schmi] G. Schmithüsen. An algorithm for finding the Veech group of an origami. To appear in Experimental Mathematics.

[Schmo] M. Schmoll. On the asymptotic quadratic growth rate of saddle connections and periodic orbits on marked flat tori. Geom. Funct. Anal. 12:3 (2002), 622-649.

[Th] W. P. Thurston. On the geometry and dynamics of diffeomorphisms of surfaces. Bull. Amer. Math. Soc. (N.S.) 19:2 (1988), 417-431.

[Ve82] W. A. Veech. Gauss measures for transformations on the space of interval exchange maps. Ann. of Math. (2) 115:1 (1982), 201-242.

[Ve87] W. A. Veech. Boshernitzan's criterion for unique ergodicity of an interval exchange transformation. Ergodic Theory Dynam. Systems 7:1 (1987), 149153.

[Ve89] W. A. Veech. Teichmüller curves in moduli space, Eisenstein series and an application to triangular billiards. Invent. Math. 97:3 (1989), 553-583.

[Ve92] W. A. Veech. The billiard in a regular polygon. Geom. Funct. Anal. 2:3 (1992), 341-379.

[Ve95] W. A. Veech. Geometric realizations of hyperelliptic curves. Algorithms, fractals, and dynamics (Okayama/Kyoto, 1992), 217-226, Plenum, New York, 1995.

[Vo] Ya. B. Vorobets. Planar structures and billiards in rational polygons: the Veech alternative. Russ. Math. Surv. 51:5 (1996), 779-817.

[Wa] C. C. Ward. Calculation of fuchsian groups associated to billiards in a rational triangle. Ergodic Theory Dynam. Systems 18:4 (1998), 1019-1042.

[Zo] A. Zorich. Square tiled surfaces and Teichmüller volumes of the moduli spaces of abelian differentials. Rigidity in dynamics and geometry (Cambridge, 2000), 459-471, Springer, Berlin, 2002. 
IML, UMR CNRS 6206, Université de la MÉditerranée, Campus de Luminy, Case 907, 13288 Marseille Cedex 9, France.

E-mail address: hubert@iml.univ-mrs.fr

Irmar, UMR CNRS 6625, Université de Rennes 1, Campus Beaulieu, 35042 Rennes Cedex, France;

I3M, UMR CNRS 5149, Université Montpellier 2, Case 51, Place Eugène Bataillon, 34095 Montpellier cedex 5, France;

IML, UMR CNRS 6206, Université de la MÉditerranée, Campus de Luminy, Case 907, 13288 Marseille Cedex 9, France.

E-mail address: samuel.lelievre@polytechnique.org

URL: http://carva.org/samuel.lelievre/ 